\title{
Surface impacts and collisions of particle-laden nanodrops
}

\author{
Joel Koplikł \\ Benjamin Levich Institute and Department of Physics \\ City College of the City University of New York, \\ New York, NY 10031
}

(Dated: April 20, 2022)

The surface impact and collisions of particle-laden nanodrops are studied using molecular dynamics computer simulations. The drops are composed of LennardJones dimers and the particles are rigid spherical sections of a cubic lattice, with radii about $11 \mathrm{~nm}$ and $0.6 \mathrm{~nm}$, respectively. Uniform suspensions of $21 \%$ and $42 \%$ particle concentrations and particle-coated drops are studied, and their behavior is compared to that of pure fluid drops of the same size. The relative velocities studied span the transition to splashing, and both wetting/miscible and non-wetting/immiscible cases are considered. Impacts normal to the surface and head-on collisions are studied and compared. In surface impact, the behavior of low-density suspensions and liquid marble drops is qualitatively similar to that of pure liquid, while the concentrated drops are solid-like on impact. Collisions produce a splash only at velocities significantly higher than in impact, but the resulting drop morphology shows a similar dependence on solid concentration as in impact. In all cases the collision or impact produces a strong local enhancement in the kinetic energy density and temperature but not in the particle or potential energy densities. Mixing of the two colliding species is not enhanced by collisions, unless the velocity is so high as to cause drop disintegration.

*jkoplik@ccny.cuny.edu 


\section{INTRODUCTION}

The collisions and surface impacts of liquid drops have been studied systematically for over a century [1 [5], with motivations ranging from the esthetics of a splash to understanding of the underlying fluid mechanics to applications such as printing and sound generation in rainfall. More recently interest has expanded to drops of complex liquids [6 10], and in particular to drops containing solid particles [11-15], which is the focus here. In recent papers we have used molecular dynamics (MD) simulations [17, 18] to study the impact of liquid drops on surfaces of different wettabilities, textures and shapes. Such calculations are naturally restricted to fairly small drops and limited time intervals, tens of nanometers and nanoseconds, respectively, but remain relevant for a number of reasons:

- MD results are controlled by the choice of atomistic interactions alone, independent of other assumptions.

- As shown in [18], in most cases the MD results are consistent with experiment and continuum calculations using straightforward modeling choices. The principal exceptions occur when the surface attraction is weak, where MD is perhaps more reliable anyway, and at higher Reynolds and Weber numbers, where MD velocities are so high as to cause disintegration.

- Nanodrops have their own potential applications, to heat transfer in micro- and nanofluidic systems. More generally the latter field is so new that is is worthwhile to explore how its small characteristic length and time scales affect familiar continuum phenomena.

As a direct continuation of our previous work we begin by asking how the presence of suspended solid particles alter the qualitative behavior of an impact with a planar solid surface. Three types of drop are considered: a low-concentration uniform suspension, a higher-concentration suspension and a liquid marble consisting of a drop decorated by solid particles on its boundary. The solid particles are rigid spherical sections of a cubic lattice, and the fluid consists of Lennard-Jones dimers. In the first case, we present results for a suspension with $21 \%$ solids concentration, where the percentage refers to the fraction of solid atoms. This case is found to have the qualitative behavior as lower concentrations since, as we shall see, the particles simply advect with the fluid during the impact. Significantly 
higher concentrations of solid do change the behavior in impact, and we discuss a $42 \%$ solid concentration drop as an example. Here the aim is to present a comparison and an illustration of different behavior, but we do not claim that the specific results hold for all high concentrations nor for all choices of the solid-liquid interaction. The third system considered here is a liquid marble in which the particle reside on the surface of a drop. In MD calculations the difference between the marble and suspension drops lies in the strength of the attraction between solid and liquid atoms, as discussed below. Many other cases are possible (and equally amenable to calculation) since drop and particle sizes, interaction form and strength, temperature, surface characteristics, etc., can all be varied, but we have limited this paper to three hopefully representative cases.

After discussing impact on solid surfaces, we turn to the head-on collisions of liquid drops and the same three suspension drops as above. Drop collisions constitute a well-studied and significant subject in itself, with numerous laboratory experiments and an extensive modeling literature; see, for example, [19 23. More recently, an MD simulation literature on collisions has begun to appear [24-29] as well, but here our principal motivation is to compare the two processes and elucidate the effects of particulate content on the interactions between deformable objects and with rigid surfaces. (An earlier paper [30] makes a similar comparison for the case of droplets residing on a solid surface, but the focus is somewhat different.) In addition, we have the practical motivation that an MD impact simulation is very easily converted to a collision simulation by simply removing the solid surface and duplicating the drop, so the computation and analysis are nearly identical. Once again the number of possible variations in collision parameters is immense and here we focus on the same three comparison cases only.

The outcome of a surface collision naturally depends on the wettability of the liquid and solid involved, and here we consider the extreme cases of complete wettability (strong attraction between solid and liquid atoms) and non-wettability (no attraction and just a short distance repulsion). The wettability of the solid particles inside the drop matters as well, but we wish to consider suspensions that are stable in equilibrium, which requires an attractive interaction. Likewise, collisions between drops of the same material are not the same as heterogeneous collisions, so we also consider the contrasting case of collisions between drops of two immiscible liquids. 
Some aspects of the outcome of a surface impact or collision are evident in snapshots of the intermediate and final states, but further understanding may be obtained from the internal density, temperature, pressure and energy density fields. In an MD simulation, any field expressible in terms of atomic variables may be calculated, and we do so here in order to understand how a drop's initial translational kinetic energy is either distributed within itself or lost to the exterior. Two related issues are the coherence of the drop - the extent to which the solid particles and liquid stay together during and after impact, and the degree of mixing between the contents of the two colliding species and in particular the degree to which it is enhanced by a hard contact. These question are discussed below in terms of the time-dependent species density fields.

The organization of the paper is as follow. A description of the MD procedure, which very closely resembles that in the previous papers [17, 18], in given next, followed by two sections describing the results of impact and collision simulations, respectively, focusing on a qualitative comparison of shape change. Next, we present a more quantitative analysis of the energy transfer where we analyze the local potential and kinetic energy and temperature fields as a function of time. A similar study of the density fields for the individual atomic species is then the basis for a discussion of the degree of mixing resulting from drop impact, and is followed by our conclusions.

\section{MD METHODS}

We are interested in spherical drops of a generic uncharged Newtonian liquid containing solid spherical particles distributed uniformly throughout the interior (suspensions) or on the surface (marbles). Since the drops are nanometer sized and subject to strong thermal fluctuations the adjectives "spherical" and "uniform" are necessarily approximate. The liquid is composed of Lennard-Jones dimers, which have the potential energy functions

$$
V_{L J}(r)=4 \epsilon\left[\left(\frac{\sigma}{r}\right)^{6}-c\left(\frac{\sigma}{r}\right)^{12}\right]+\delta V(r) \quad V_{F E N E}=-\frac{1}{2} k r_{0}^{2} \log \left(1-r^{2} / r_{0}^{2}\right)
$$

The Lennard-Jones (LJ) potential $V_{L J}$ acts between all atoms within a cutoff distance $r_{c}=$ $2.5 \sigma$, and the potential is shifted by a linear term $\delta V$ so that the force vanishes at the cutoff. The constant $c$ in $V_{L J}$ adjusts the strength of the attractive interaction; its value is unity for fluid atoms of the same species and for fluid-solid interactions for a liquid which completely 
wets a solid surface, but is zero for the interaction between atoms of "immiscible" drops and for the fluid-solid interactions of a completely non-wetting liquid. The fluid-particle atomic interaction coefficient is again unity for a suspension, where the fluid completely wets the particles which then prefer the interior of the drop, but we use a weaker value $c=0.75$ for the marble case so that the particles are less attracted and prefer the drop surface. This specific numerical value was chosen because it produces a $90^{\circ}$ contact angle for a drop on a planar solid surface. The FENE (finitely extensible linear elastic) potential acts between each pair of atoms in a dimer molecule. Dimers are used in place of monomers because the monatomic Lennard-Jones system has a very high vapor pressure, making it difficult to identify the liquid-vapor interface. The resulting vapor pressure under the operating conditions of the simulations is roughly half an atmosphere. In a previous paper [17] we considered tetramer molecules as well, which have negligible vapor pressure, and showed that in contrast to macroscopic drops [32] the presence of vapor is irrelevant in nanodrop surface impacts. The results here pertain to dimers but the general characteristics of their behavior is expected to be general. When we discuss variations in the local energy density below, the explicit numbers refer to the sum of the LJ and FENE energies in Eq. 1; we are concerned only with energy variation within the drops so that the choice of the zero of energy is irrelevant.

In the remainder of the paper lengths are given in terms of the approximate LJ atomic diameter $\sigma$ and times in terms of the natural time unit $\tau=\sigma(m / \epsilon)^{1 / 2}$ where $m$ is the common mass of all atoms. Energies are expressed in term of $\epsilon$ and temperatures in terms of $\epsilon / k_{B}$, where $k_{B}$ is Boltzmann's constant. For physical estimates of dimensionful quantities we use LJ parameters for argon, $\sigma \approx 0.34 \mathrm{~nm}, \tau \approx 2 \mathrm{ps}$ and $\epsilon / k_{B} \approx 120^{\circ}$.

Fluid atoms move according to Newton's equation with a force given by the gradient of the potentials above, integrated by Gear's method with a time step of $0.0005 \tau$, a relatively small value to allow for the significant collisional velocities. The particle motion is found by computing the net force exerted on the particle's atoms by neighboring fluid atoms, and integrating Newton and Euler's equations for rigid bodies, in quaternion variables [33.

To prepare the drops, we begin with a cubic lattice of atoms, and first select all atoms within a radius $36 \sigma$ of a center. Within this spherical region, initial particle centers are located at uniformly distributed sub-lattice sites, and atoms with a radius $2 \sigma$ of each center are assigned to a particle. The resulting particles contain 32 atoms each and have a rather 


\begin{tabular}{|c||c|c|c|c|}
\hline & liquid & $21 \%$ & $42 \%$ & marble \\
\hline \hline fluid atoms & 157216 & 106638 & 90770 & 125470 \\
\hline particles & - & 1048 & 2043 & 649 \\
\hline radius & 36.0 & 34.9 & 36.2 & 35.0 \\
\hline
\end{tabular}

TABLE I. Drop parameters

ragged appearance when each atom is depicted as a sphere, but an examination of the time-averaged liquid density field around a particle in equilibrium shows that the effective particle is in fact spherical with a radius of approximately $2 \sigma$. For liquid marbles, the particle centers are instead located at uniformly spaced positions on the surface of the fluid sphere. The composite drop is placed at the center of a periodic box of dimensions $(X, Y, Z)=$ $(300,100,300) \sigma$, and initially given random velocities normalized to a temperature in the solid phase, $T=0.2$. The temperature is then ramped up into the liquid-vapor coexistence region at $T=0.8$ over a time $100-300 \tau$, using a Nosé-Hoover thermostat. This ramp procedure allows the drop to melt into a well-define liquid sphere surrounded by vapor without violent shape fluctuations. Subsequently, the drop is equilibrated at $T=0.8$ until the shape and the average particle distribution stabilize, typically requiring $500-1000 \tau$.

The structure of the drops is displayed in Fig. 1, where for each case we show a snapshot of a slab of width $10 \sigma$ through the center along with the total, fluid and particle radial densities $n(r)$, at the end of the equilibration period. The particles in the two suspension drops are approximately uniformly distributed but those in the liquid marble are not, and show some unavoidable spatial variation along the surface. The reason is that the marble particle atoms must have a weaker attraction to the liquid to remain on the drop surface and are consequently more mobile. The only other variable parameter is the surface density of particles, but at lower density they are even more mobile while at higher density they tend to leave the surface. The number of fluid atoms and (32 atom) particles in each drop is given in Table I, along with the nominal radius, defined as the point where the total density falls to half the value at the center. We also indicate the number of atoms and radius of a pure liquid dimer drop used for comparison below. The drop diameters and number of atoms are similar in all cases, but not precisely the same.

For surface impact simulations, a denser slab of $f c c$ solid, with lateral dimensions $(X, Z)=$ 


\begin{tabular}{|c||c|c|}
\hline$u_{0}$ & $R e$ & $W e$ \\
\hline \hline 1.0 & 10.3 & 56.5 \\
\hline 2.0 & 41.2 & 226. \\
\hline 3.0 & 31.0 & 508. \\
\hline
\end{tabular}

TABLE II. Flow parameters.

$(300,300) \sigma$ and one unit cell thickness in $Y$, containing 147,456 atoms, is placed at the base of the simulation box. These solid atoms are tethered to their lattice sites using a spring of stiffness $100 \epsilon / \sigma$, allowing small thermal fluctuations in position. The solid is equilibrated at the same temperature $T=0.8$ as the equilibrated fluid. Impact is initiated by turning off the fluid thermostat and giving each atom in and near the drop a fixed downward velocity. The solid temperature is fixed throughout the simulation by the thermostat, corresponding physically to impact on a material of very high thermal conductivity held at fixed temperature. For drop collision simulations, after the fluid has equilibrated, the box is doubled in size and a duplicate atomic system with the same velocities and relative positions is placed in the open region just above the original drop. A further (short) equilibration interval allows the vapor in the region between the drops to mix uniformly, and then the two drops are given equal and opposite velocities so that they collide. The latter instant is defined as $t=0$ in the subsequent results.

The Reynolds and Weber numbers for the liquid drop at the collision velocities $u_{0}$ studied in this paper, $R e=\rho u_{0} R / \mu$ and $W e=\rho u_{0}^{2} R / \gamma$, are given in Table II. The viscosity $\mu=$ $2.80 \mathrm{~m} / \sigma \tau$ and the surface tension $\gamma=0.51 \mathrm{~m} / \tau^{2}$, of the pure fluid are determined in separate simulations of Couette flow and a planar liquid vapor interface for the same liquid drop, as discussed in [17]. The latter paper calculates the sound speed for the dimer liquid studied here, $c_{s} \approx 4 \sigma / \tau$, and the resulting Mach number $M a=u_{0} / c_{s}$ ranges from 0.25 to 0.8 . The presence of particles of course alters the viscosity and surface tension of the drops, so these numbers should be thought of as convenient non-dimensionalization of the velocity but not exactly the ratios of accurate physical length or time scales.

In the remainder of the paper we display the results of only a single simulation for each value of particle concentration and impact velocity, but in fact a number of additional cases with small differences in drop size, velocity and interactions were carried out as well. The 
differences preclude simple averaging of the results, and in any event there are too few runs available to constitute a reliable statistical sample, but the qualitative results described below here are robust in the sense that the behavior is not significantly altered if a different realization of the initial conditions or a small variation in parameters is considered.

\section{SURFACE IMPACT}

In this section we discuss the impact of particle-laden drops on atomistic solid surfaces which are homogeneous, planar up to thermal fluctuations and are either completely wetting or completely non-wetting to the liquid and solid atoms within the drop. For reference we recall the behavior of pure liquid drops [17]; a snapshot of the two wettability cases at time $100 \tau$ is shown in the left-hand columns of Fig. 2 and Fig. 3, respectively, for different initial velocities. At $u_{0}=1.0 \sigma / \tau$ the drop deforms and spreads laterally after impact without emitting significant vapor. At later times, a drop on a non-wetting surface contracts, lifts off the surface and eventually regains its spherical shape, whereas on a wetting surface the drop after its initial deformation continues to spread but at a much slower rate. At $u_{0}=2$ the drop initially forms a crown splash and subsequently disintegrates, producing extensive vapor and leaving a ramified pattern resembling spinodal decomposition in the non-wetting case but a fairly well-defined circular puddle on a wetting surface. At $u_{0}=3$ the drop disintegrates immediately and fills the simulation box with vapor, while the surface is left with tendrils of liquid in the non-wetting case or an irregular puddle on a wetting surface. At times later than shown in the latter two cases, the vapor becomes uniformly distributed in space (the simulation domain is a periodic box) while the liquid remaining on the surface aggregates into larger droplets.

An example of the time evolution of the impact of a particle-laden drop is given in Fig. 4, for the case of the $42 \%$ suspension falling on a non-wetting surface at $u_{0}=2 \sigma / \tau$. (Analogous plots for pure liquid drop impact are given in [17].) After contacting the solid surface a fluid rim forms $(20 \tau)$, and then a lamella of fluid and isolated single particles proceeds radially outward along the surface, accompanied by a crown splash $(35 \tau)$. The lamella breaks into fragments and particles begin to cluster $(50 \tau)$ and then the body of the drop breaks up $(75 \tau)$. At $100 \tau$ the fragments begin to coarsen, and subsequently the solid structure becomes smoother while the fragments move off. While the time evolution of the 
drop differs in detail from case to case, we note that the fate of the drop is evident at time $100 \tau$, so we summarize the results of the various impact simulations by displaying their state at this time, in Figs. 2 and 3 for now-wetting and wetting surfaces, respectively.

The behavior of the $21 \%$ concentration suspension is rather similar to that of pure liquid, the principal differences being a reduction in the degree of lateral spreading, and a more attenuated surface residue of liquid along with more vapor in the higher-velocity impacts. At later times, the low velocity drop recoils from the surface and becomes spherical while the other cases continue to produce vapor while their surface residue coarsen. The term "coarsening" here is used in further analogy to spinodal decomposition and indicates that the thicker liquid tendrils on the surface accumulate molecules and particles and thicken while the thinner ones evaporate. The reduction in the spreading diameter may be attributed to the increased viscosity of a suspension in comparison to pure liquid. The additional vapor and stringier surface residue at the higher impact velocities, in comparison to pure liquid, we attribute to the clustering of liquid around the particles, whose atomic interactions with the liquid have the same strength as the liquid-liquid ones in this case.

The higher concentration (42\%) suspension shows distinctly different behavior. At low velocities a nearly rigid bowler hat shape is formed, in which the solid particles first striking the surface are directed outwards and begin to spread laterally but the bulk of the drop acts as a semi-rigid body and retains its original shape. In fact the lateral motion ceases at around time $70 \tau$, while the drop deforms the solid surface downward, and on recoil the drop leaves the surface with little further change in shape. Similar behavior is observed in experiments involving the impact of drops of yield-stress fluids [7, 8]. At a higher velocity, $u_{0}=2.0 \sigma / \tau$, the drop is strongly deformed into an irregular and partially fragmented saucer shape, with less vapor and more liquid retained in the saucer. The particles in a sense act as a glue holding the drop together. The drop breaks up completely only at the highest velocity, leaving a surface residue in the form of small suspension clusters. At later times the higher velocity impact states coarsen in part: smaller fragments of solid become smoother while remaining in place or else merge with the larger fragments, while the larger ones grow in volume while becoming more compact as they accumulate liquid atoms and smaller solid clusters.

We have also considered the situation where there is no attractive potential between the solid atoms in different particles and only a short-distance repulsion. At low impact velocity 
$u_{0}=1.0 \sigma / \tau$ the particles move with the fluid, and the result resembles the $21 \%$ case, but at higher velocities the particles shoot out horizontally and accumulate at the edges of the simulation box, so that the drop's disintegration is more violent than at lower particle concentration. A recent experiment observed the ejection of a monolayer of particles in the surface impact of a suspension drop [15], but in these simulations several particle layers are seen. The contrasting behavior may originate from different interactions as well the size difference, but we do not pursue the matter here.

The liquid marble's behavior is roughly intermediate between fluid and $21 \%$ suspension, both in spreading diameter at low velocity and in the amount of vapor produced in faster impacts. The impact tends to dislodge the particles from the drop surface leaving them isolated, because in this case the atomic interactions particle and liquid atoms are weaker than those of the liquid. On the whole, the particles make little difference to the behavior of the drop in impact, and the slightly smaller amount of vapor and surface tendrils generated is the results of having fewer liquid atoms in the original drop.

The $100 \tau$ state of the same set of drop impacts on a wetting surface is shown in Fig. 3 , At the lowest velocity there is an obvious difference in the shape of the rim at the surface: the drop atoms are now pulled towards the surface to produce a downward rather than upward-facing curvature. Otherwise, all four drop shapes and lateral extent are similar to those on a non-wetting surface. (See [18] for a discussion of low velocity impacts on intermediate wettability surfaces.) When these cases are continued to longer times, the liquid spreads slowly while the particle-laden drops show negligible change in shape. A second qualitative difference with respect to a non-wetting surface is that in all of the higher-velocity impacts a residual circular saucer-shaped liquid puddle remains on on the surface. Both differences simply reflect the attraction of the surface atoms, absent in the non-wetting case. Furthermore, this attraction suppresses the formation and coarsening of the spinodal-like liquid tendrils seen in that situation, by acting to hold the liquid in place. For either type of surface, at higher impact velocity the particles form smaller droplets mixed with liquid at low concentration, tend to disperse in the marble case because of their weaker attraction to the liquid, and form nearly rigid irregular clusters at high concentration. At times beyond that shown in the figure, the puddles persist and become somewhat more regular, the vapor tends to becoming uniform in space, and in the concentrated suspension the solid clusters tighten up and exhibit some smoothing at the extremities. 


\section{DROP COLLISIONS}

A head-on collision of two drops has a superficial similarity to a normal collision on a solid surface, in the (continuum) sense that one might imagine that the colliding drops should be mirror images of each other with the mid-plane playing a role similar to that of a reflecting boundary. In reality, the atoms in a solid surface respond to those of an incoming liquid drop in a rather different manner than those in a second drop, and it is of interest to compare the two processes. We first consider the collisions of two drops which are "identical" in an average sense, which is to say they have the same number of atoms and were prepared (equilibrated) in the same way. The two drops would then have the same density, mean radius, interfacial thickness, etc., but distinctly different atomic positions and momenta. In practice we cheat slightly: we duplicate a single equilibrated drop and translate it to a position just above the original, and then shift the atomic velocities in the two drops by equal and opposite amounts so as to make them collide. Note that the top and bottom of the original drop have relative positions and velocities which differ in detail (although they have the same average) and it is these which control the initial collision process. If instead we had reflected the original drop configuration about a mid-plane before shifting velocities the collision would have involved two unrealistic mirror image drops.

The results of collision simulations of the four types of drop whose surface impacts were considered in the previous section is shown in Fig. 5, at the same three relative velocities. When two identical pure liquid drops collide (first column) their respective atoms attract each other and the drops merge. At relative velocity 1.0 and $2.0 \sigma / \tau$ the drops merge smoothly: once the drop's atoms are within interaction range a neck forms and thickens, the drops continue to move towards each other and combine into a peanut shape which subsequently evolves into a prolate ellipsoid, which in turn eventually relaxes to a (larger) sphere under the action of surface tension. In this case, and also if the solid concentration is not too high, there is a decaying oscillation about the final spherical shape. The effect of increasing the initial relative velocity is to increase the initial compression giving a disklike intermediate state. When the initial velocity is further increased to $3 \sigma / \tau$ the disk is thinner and becomes rather ragged at its edges; in this simulation it reaches the edge of the simulation box and effectively merges with its periodic neighbors, but in open space it would emit small secondary droplets from the rim while the main body eventually contracted to a 
sphere. At still higher collision velocities (not shown) a merged disk forms but spreads so rapidly as to open holes and then disintegrates into smaller drops. These collisions may be compared to impact on a wetting (attractive) surface: there is a visual similarity between half of the lower-velocity collision shapes here and the impacting drop at velocity $1.0 \sigma / \tau$, but the variation of drop radius and height with time is not the same. More significantly, an impact at velocity 2.0 causes a drop to disintegrate whereas in collisions the drop retains its integrity even at relative velocity 3.0. Physically, atoms in a solid are constrained to have small displacements and tend to cause impinging liquid molecules to reflect and transfer their kinetic energy back to the incident drop, whereas atoms in a second drop are not so constrained and are capable of absorbing and redistributing the incident energy. A more detailed analysis of energy transfer is given in the next section.

The same collision protocol for the various particle laden drops gives the states at time $100 \tau$ shown in the remaining columns of Fig: 5. The $21 \%$ suspension drops merge smoothly at the lowest collisional velocity but at a much slower rate, going from touch to peanut to sphere (in the figure) to slightly prolate ellipsoid (later), and continues to contract at $1000 \tau$. Relaxation to the final spherical shape requires much longer times. At velocity $2 \sigma / \tau$, the behavior is similar but the time scale differs: the drops merge and contract into an ellipsoid, and then begin to relax back after $150 \tau$, but remains highly ellipsoidal even at $1000 \tau$. At both of these impact velocities, the particles remain uniformly distributed in the drops' interiors. At initial velocity $3 \sigma / \tau$, however, a thin disk of merged liquid forms having an irregular rim and with some particle and secondary droplet emission. Subsequently, the edges smooth due to rearrangement and the drop begins to relax to a sphere, but very slowly and again remains highly disk-like at $500 \tau$. Snapshots of the detailed time evolution for this case are shown in Fig. 6. At still higher collision velocity a thin disk with irregular edges forms, which again develops holes as it expands to each the box boundary and emits small particle clusters.

The $42 \%$ suspension again shows a semi-solid behavior. At incident velocity $1.0 \sigma / \tau$ a partial merger occurs in the contact region, leading to a peanut shape after about $70 \tau$, which shows almost no subsequent shape variation aside from a very weak contraction. More precisely, if we examine the location of the center of mass of the original liquid and solid atoms in the two drops, after colliding the average position of the solid atoms hardly changes, whereas that of the liquid atoms contracts very slowly $(\sim \pm 0.05 \sigma / \tau)$ as they rearrange inside 
the solid structure. At velocity $2 \sigma / \tau$ the original drops move closer together after contact than in the previous case, leading to a rough oblate ellipsoid, but again nearly freeze in place after $70 \tau$. Here the collision produces an outgoing liquid sheet in the collision plane, which breaks up into droplets. A small rim of particles is carried along and several additional small particle clusters are emitted. At the higher velocity $3 \sigma / \tau$, the same behavior is more evident, with a stronger emission of particle and fluid along the mid-plane, leaving an irregularlyshaped nearly-rigid suspension drop behind. A simulation at still higher velocity $4 \sigma / \tau$ gives a qualitatively similar result, with more emission and irregularity.

The liquid marble collisions at low velocity $1.0 \sigma / \tau$ allow the particle to remain mostly at the interface, where they have the effect of squeezing the liquid into a thinner and broader disk than a pure liquid drop, presumably due to their inertia, although some particles are knocked loose by the collision. At higher velocities many particles are dislodged from the surface of the drop, and their outward motion broadens and distorts the edges of the liquidfilled disk at $u_{0}=2 \sigma / \tau$ and tears it apart completely at velocity $3 \sigma / \tau$. A recent experiment involved the collision between a moving, densely-covered liquid marble and a second, much larger one at rest [16]. At low velocities the moving marble separates after impact while at higher velocites it is absorbed. However, the system studied is somewhat different from the present simulations; aside from the degree of surface coverage, the experimental marbles are large enough for gravity to play a role in their behavior.

Just as collisions of drops of the same material has a superficial similarity to impact on a wetting surface, we can compare impacts on non-wetting surfaces to collisions between drops of "opposite" material by choosing the atoms of the respective drops to have only a repulsive interaction. In practice we set the coefficient $c$ in Eq. 1 to zero for interactions involving both fluid and particle atoms in the different drops, and repeat the same suite of collision simulations as above. The results, again at time $100 \tau$, are given in Fig 7. The obvious qualitative difference is that the drops stand each other off rather than merge, and even at the highest velocity when the drops disintegrate, the fragments mostly occupy their original half-spaces. A concrete illustration of the collision process for this case is given in Fig. 8 for the case of two "opposite" marbles at relative velocity $2 \sigma / \tau$. Note that the particles remain attached to the drops until they become severely deformed, and even then only a few are expelled, a quite different behavior from that of a surface impact at this velocity. In most cases the structure after collision has the same general stability as collisions between drops 
of the same material in terms of the smoothness of the drop/vapor boundary and the degree to which particles are expelled. The principal differences are seen in the mid-plane contact region, which we discuss further in the next section.

\section{ENERGY TRANSFER}

The translational kinetic energy of a drop is redistributed between the drop and the surface on impact or between the two drops in a collision, but the details of the division between kinetic and potential energy within the $\operatorname{drop}(\mathrm{s})$ and energy transferred to the solid is not obvious. Since the simulations track the atomic positions and momenta, the relevant information is available, and in this section we study the local distribution of energy. Since both normal surface impacts and head-on collisions of spherical drops have an ostensible rotational symmetry about the collision $(y)$ axis, we adopt a cylindrical coordinate system $(r, \phi, y)$, average all fields over the azimuthal angle $\phi$ and consider the energy fields as a function of "height" $y$ and two-dimensional radius $r$ at different times, separately for fluid and particle species. In Fig. 9 we illustrate the coordinate system and the surface elevation format for the subsequent field plots, using the density field $(d)$ for a pure liquid drop in equilibrium as an illustration. Aside from the energy, since we remarked above that the Mach number in these simulations is not small and fluid compressibility may play a role, the spatial variation of density on impact is likewise of interest. Furthermore, the individual species densities provides information on the coherence of fluid and particles and the degree of mixing of the species in the collision case, so in the next section we present the time evolution of the individual species density fields as well. In fact the axial symmetry is approximate at best, and absent in all but a statistical sense in the higher-velocity impacts and collisions, but the fluctuation information lost in this way is of lesser interest. In this section, we give several examples of the evolution of various internal flow fields within the drop and then draw some general conclusions.

We begin with a representative surface impact case, a $42 \%$ suspension drop incident on a non-wetting surface at velocity $u_{0}=2 \sigma / \tau$. in Fig. 10 we present the cylindricallyaveraged total density, potential energy, kinetic energy and temperature fields, during the earlier stages of the impact. Corresponding snapshots are given in Fig. 4. The density field exhibits fluctuations and a slight rise at the top of the drop where fluid accumulates at first 
and is later expelled into vapor, but no systematic variation in the interior and, in particular, only a slight $20 \%$ transient enhancement at the bottom where it hits the solid surface. The potential energy roughly follows the density and, somewhat surprisingly for a hard collision, shows only a similar rise at the solid surface just after contact. The subsequent decay is due to the dispersal of fluid into vapor, leaving the particles behind in the irregular saucer seen in the snapshot. The ridge in the potential energy field at the edge of the drop appears because the atoms at the edge have fewer interacting neighbors and carry less (negative) LJ interaction energy, leading to an increase in the value of the energy density there.

The effects of the impact are most apparent in the kinetic energy. The fluid kinetic energy carried by the drop initially builds up at the impacting surface and is then carried outward along it by the spreading fluid lamella before dispersing, partly to the remainder of the drop and partly to the solid surface where it is conducted away (i.e., removed by the wall thermostat). The particle kinetic energy is small near the surface immediately after impact but subsequently a packet of energetic particles move outward along the surface as they are displaced to form the solid saucer seen in the snapshots, and eventually dissipates as the particles come to rest. The local fluid temperature (the particles are athermal) also rises near the surface, beginning at impact and persisting throughout the time interval shown in the figure. The persistent enhancement in temperature near the surface is the origin of the disintegration of liquid drops in impact at this velocity [17]. Note that although the translational kinetic energy is dissipated into the vapor and the thermostat, the temperature is the fluctuation in kinetic energy about the local mean, which decays due to the diffusion of momentum on a longer time scale.

The field plots for the other drop impact cases, as well as those for a drop impact on a wetting surface, are similar in that the qualitative description in the previous paragraph is equally applicable. The one distinction is that for smaller impact velocity the temperature and kinetic energy increases are smaller, and for higher velocity the increases are larger. However, interesting differences do appear in the analogous plots for drop collisions, shown for two examples in Fig. 11 and 12 for both drops of the same material (21\% suspension at $\left.u_{0}=3 \sigma / \tau\right)$, and opposite or non-attracting materials (marbles at relative velocity $u_{0}=$ $2 \sigma / \tau)$, respectively. Just as in the impact example just discussed, the trends and general behavior of the potential energy plot follows that of the atomic density while the fluid kinetic energy follows the temperature, so we focus on the three energy plots. In collisions of drops 
of the same material, there is a smooth merger of the drops into a disk, with a small transient rise in potential energy in the merger region but a persistent ridge in the fluid kinetic energy which propagates outward along the mid-plane. The total potential energy is increased by the collision but shows little change subsequently: $6.27 \epsilon$ per particle in equilibrium to 7.31 just after contact to 7.66 after $500 \tau$. (The energy is computed as the sum of negative LJ energy and positive FENE energy, and the latter happens to have a larger magnitude.) The fluid temperature is $0.8 \epsilon / k_{B}$ in equilibrium and, similarly, stabilizes at 0.94 at the end of the simulation. The particles all have an initial translational kinetic energy but after colliding most come almost to rest except for those at the edge of the merged drop which move outward to form a saucer before halting. In the case of opposite materials, the fluid energies dip at the mid-plane reflecting the gap between the colliding drops, but the energy distribution is otherwise similar to the previous case.

\section{MIXING}

The drops considered here have particles roughly uniformly distributed during the equilibration stage, but given the contrast in mass between the fluid molecules and the solid particles, an impact may produce some relative motion and inhomogeneity. Our analysis is based on computing the probability distribution for the various atomic species as a function of the coordinate $y$ along the impact axis. We begin with the $21 \%$ suspension at low impact velocity $u_{0}=1.0 \sigma / \tau$ on a non-wetting wall, shown in Fig. 13 . The particle and fluid distributions overlap well just after impact, at maximum spreading and in the early stages of withdrawal from the wall, indicating that uniform mixing is preserved. Even at the higher velocity $u_{0}=3 \sigma / \tau$ where the drop disintegrates, fluid and particles remain well mixed: the peaks in the respective distributions always overlap, although there is a higher probability for vapor to occupy the space above the drop. Impacts on a wetting wall likewise show little separation of liquid and particles, although in this case there is always a liquid layer adjacent to the wall. In the denser $42 \%$ suspension the behavior is generally similar except for a tendency for liquid to shoot out along the wall ahead of the particles at early times, although the distributions overlap well afterwards. Different behavior is seen in the liquid marble case in Fig. 14. here the particle atoms are relatively weakly bound to the drop and are more prone to separate. The particles shoot out just after impact and are preferentially 
located near the surface, while at later times after bouncing off the surface they tend to lie above the liquid. The figure illustrates this behavior for the more dramatic case $u_{0}=3 \sigma / \tau$ but the same behavior is seen at lower velocities. We conclude that as long as the particle atoms are strongly attracted to those of the liquid (in continuum terms, the liquid wets the particles), uniform mixing is preserved in surface impacts.

A similar analysis of the relative motion of liquid and solid is relevant in drop collisions, and in addition there is the related question of whether there is any enhancement in the rate or degree of mixing of the materials in the two colliding drops. In particular, we wish to contrast the behavior of colliding drops to that of (quasi-static) coalescence where two drops are simply placed in close proximity and allowed to merge spontaneously. To this end, we have also simulated free coalescence by taking the initial configuration of two liquid drops used in the collision calculations and shifting the respective drop atomic positions towards each other so that the tails of their density profiles just overlap. The system was then allowed to evolve freely, with no imposed relative velocities of the drops and no thermostat acting, and after a short transient period the two drops merged smoothly. The evolution of the density pdf as a function of $y$ is shown in Fig. 15 for time up to 500 $\tau$ : the increasing overlap of the two original distributions reflects the degree of mixing of the drops. The effect of collisions is to increase the speed of this process, as shown in Fig. 16. When the drops collide with relative velocity $1.0 \sigma / \tau$, after $500 \tau$ the overlap region is twice as wide, even though the drop centers are slightly farther apart. The latter property results from the fact that the collision initially squeezes the drops close together, followed by a partial recoil, and then a decaying oscillation at longer times. At twice the collision velocity, the oscillation is even stronger but at the same time $500 \tau$ the distribution overlap is much greater. At still higher velocity, however, the collision produces a thin sheet which develops holes and reaches the edge of the simulation box at $150 \tau$, preventing recoil. Nonetheless, by this time the $y$-distributions overlap almost completely and the molecules of the original drops are very well mixed. The degree of mixing thus increases monotonically with collision velocity.

Although not the main focus of this paper, there has been extensive recent interest in the time-dependence of the radius of the neck formed when two drops coalesce, which provides an opportunity to compare experiment and asymptotic analysis. This information is available here as a by-product of the coalescence simulation described above, and furthermore we are able to track the growth of the neck radius for colliding drops as a function of time. 
The procedure is to examine the radially-averaged density field as a function of $r$ and $y$, discussed above, and for each $y$ identify the liquid-vapor interface as the value of $r$ when the density equals half the bulk value. Coalescence is taken to begin when this interface radius develops a non-zero minimum between the drop centers, and we plot the minimum value of a function of the time $t$ after coalescence in Fig. 17. In all cases, ranging from free coalescence to violent collision at velocity $3 \sigma / \tau$, the neck radius is seen to vary as $t^{1 / 2}$ over one to $1-1 / 2$ decades in time. Not surprisingly, the growth rate itself (the prefactor) increases with impact velocity, roughly linearly. The variation is somewhat irregular at early times, due to thermal fluctuations in shape, until the neck radius reaches a value of around $10 \sigma$ (neck diameter $\sim 20$ atoms). The curves turn over in the case of colliding drops, because there is a residual oscillation after merger. We should emphasize that the behavior shown here pertains to nanodrops, where only about one decade of radius variation is available, and it is possible for other dynamical regimes to be present when larger drops merge [21, 23]. In fact, another MD simulation [29] using a different choice of interatomic potentials found a crossover between the expected linear growth law for the neck radius at early times and $t^{1 / 2}$ growth afterwards. However, we note a recent lattice-Boltzmann study [31, ostensibly relevant to macroscopic drops, found the same result as seen here, a single square-root growth law for the neck radius.

Returning to the issue of mixing, the same analysis used for liquid drops is applied to the particle-laden drops, where there are now four distributions, for the fluid and particles in each of the two initial drops. A typical example is show in Fig. 18, for the collision of identical $21 \%$ suspension drops at relative velocity $2 \sigma / \tau$. One sees the degree of mixing of the two drops increasing with time, but at a much slower rate than in the pure liquid case at this velocity. The slower motion of the relatively massive particles presumably slows the mixing process. Another feature to note is that the particles tend to stay with their original liquid, in the sense that for each drop the fluid and particles distributions are nested. The recoil of the drops after impact is also evident in this plot. The same trends are observed at both lower and higher collision velocities for this material, but different behavior is seen in the other drops. The $42 \%$ suspension drops distort when they collide, but after some weak initial mixing the $y$-distribution stabilize. An example at velocity $2 \sigma / \tau$ is given in Fig. $19 \mathrm{a}$ at $100 \tau$. After this time there is little change in the spatial distributions, and the drop's behavior is closer to that of a solid. As one would expect, the width of the 
mixed zone is broader and narrower at lower and higher collision velocities, respectively, but always stabilizes after some initial deformation. In the case of the liquid marble drop, the particle distribution no longer nests inside the fluid distribution, since the liquid-solid atomic interaction is weaker and particles are expelled after collision. Nonetheless the presence of particles still slows the mixing of the liquids, as indicated in Fig. $19 \mathrm{~b}$, for velocity $2 \sigma / \tau$ at $100 \tau$. A last variant case is the collision of "nonwetting" drops, whose respective atoms have only a repulsive interaction. Here the $y$-distributions of the atoms in the original drops remain separated after the collision, as illustrated in Fig. 19. for 21\% suspension drops at the same velocity and elapsed time.

We wish to emphasize that while while we have provided detailed information for a only subset of the simulated cases in this section, the stated conclusions concerning the qualitative aspects of energy transfer and mixing are generic, at least within the range of molecular systems studied here.

\section{CONCLUSIONS}

We have used MD simulations to study the surface impacts and collisions of nanometersized suspension drops. The range of impact velocities was chosen to span the full range of phenomena from bouncing accompanied by distortion and recovery to violent disintegration, and collision simulations at the same relative velocities provided a direct comparison of the two processes. Only normal impacts and head-on collisions were considered here, but other cases could be easily studied using the same methods. The behavior of four types of drop were compared: pure (diatomic) liquid, homogeneous suspensions of either "low" (21\%) or "high" (42\%) particle volume fractions and a "liquid marble" with a surface coverage of roughly 40\%. We have also carried out simulations for drops with lower volume fractions, but these behave in a qualitatively similar manner to the $21 \%$ suspension and marble cases presented here. Highly concentrated suspensions exhibit distinctly different behavior, however, which we have only begun to explore here.

A general conclusion is that the presence of nanoparticles in a drop, either uniformly suspended or coating the surface, does not qualitatively alter its impact behavior unless the solid concentration is quite high. In the former case, corresponding to particles which are strongly wetted by the liquid, changes in spreading radius and rate are seen but the 
behavior is that of a liquid of higher viscosity and the fate of the drop is unchanged. In the latter case, where the solid-liquid interactions are of intermediate strength and the particles reside at the surface, the nanoparticles are driven by the bulk fluid motion at low impact speeds and simply tend to fly off in more rapid impacts. For these drops the distinctions between the bouncing, splashing, disintegration, coalescence and stand-off regimes depend most critically on impact velocity. The concentrated suspension drops tend to be more coherent in impact because the nanoparticles here have a short-distance attraction to each other and in a sense act to glue the drop together. A limited analysis of the contrasting case of no direct interparticle attraction shows the opposite behavior, with the particles flying away from the impacting liquid region. More work is needed to understand the relation between the concentrated suspension drop simulations here and the flow of bulk suspension flows, where various non-linear rheological behaviors are observed.

Some further specific conclusions on mixing and energy transfer are as follows.

1. The atomic density does not increase significantly in the contact region as a result of the process. In a wetting impact or a collision of the same materials, the density gap rapidly fills but does not rise substantially in height above the bulk values before impact. In the opposite case of impact on a wetting surface or a collision between atomically repelling materials, a density gap of atomic thickness persists but there is no formation of a higher-density ridge of molecules or particles in the impact regions to either side.

2. In correspondence to the absence of an increase in density, there is no local enhancement in the potential energy.

3. The fluid kinetic energy and temperature both rise locally in the contact region, to a degree that increases with the relative velocity. Much of the kinetic energy dissipates relatively quickly into the thermostatted wall or the bulk of the drop(s) but the temperature enhancement persists over longer times. In surface impact the drop temperature eventually falls to that of the (thermostatted) wall but in collisions the drop temperature can only equilibrate relatively slowly with the low-density surrounding vapor.

4. Collisions are "gentler" than surface impacts, in the sense that significantly higher 
velocities are required to disintegrate a drop in a collision $\left(u_{0} \sim 3-4 \sigma / \tau\right)$ than in an impact $(\sim 2 \sigma / \tau)$. A rigid solid surface tends to reflect impinging molecules, causing disruptive hard interactions with those in the interior of the drop, whereas in a collision the molecules in the two drops are relatively free to rearrange themselves locally without causing global changes in the configuration.

5. Drop collisions enhance the rate of mixing of identical drops of liquid, but have only a modest effect in promoting fluid and particle mixing in the suspension case. When the materials are repelling, in the sense of having no atomic attraction, a collision does not promote mixing unless it is so violent as to disintegrate the drops.

The goal of this paper is to identify trends rather than to make quantitative predictions, since few experimental results at this scale are available and furthermore it would be difficult to precisely reproduce the simulated systems experimentally. In a previous paper [18], we were able to compare (mostly favorably) our results for low-velocity impact nanodrop simulation to experimental and theoretical scaling laws for continuum scale pure-liquid drop spreading. However, such data is not available for the particle-laden case and, in addition, in higher-velocity phenomena such as splashing the nanoscale simulations behave differently than laboratory experiments. A principal reason is that when the impact velocity is chosen to match the laboratory values of Reynolds and Weber numbers where the transition to splashing is found, the small drop size necessitates velocities so high that the Mach number becomes appreciable and the post-impact temperature rises above the liquid-vapor coexistence value. A second issue is that the small size of the drops precludes the appearance of all of the regimes observed in larger systems, which develop on longer space and time scales not present here. The obvious remedy - bigger simulations - is problematic because at least a decade in size variation is required to pin down the scaling characteristics of any flow regime, and a decade increase in drop radius is a factor of 1000 in the number of atoms. MD codes are at best vary linearly in CPU time with particle number, and furthermore bigger systems require longer physical times to develop, so this approach is impractical at present. A more promising means of connecting nano- and larger scale drop phenomena may be the use of coarse-grained fluid mechanical models, such as discrete particle dynamics, but in this case the atomic resolution is absent, and some effort would be required to reliably connect such a model to molecular interactions and structure. 
[1] A. M. Worthington, A Study of Splashes (Longmans, Green, London, 1908).

[2] M. Rein, Phenomena of liquid drop impact on solid and liquid surfaces, Fluid. Dyn. Res. 12, 61-93 (1993).

[3] A, L Yarin, Drop impact dynamics: splashing, spreading, receding, bouncing, ..., Annu. Rev. Fluid Mech. 38, 159-192 (2006).

[4] M. B. Lesser and J. E. Field, The impact of compressible liquids, Annu. Rev. Fluid Mech. 15, 97-122 (1983).

[5] M. Marengo, C. Antonini, I. V. Roisman, and C. Tropea, Drop collisions with simple and complex surfaces, Curr. Opin. Colloid Int. Sci. 16, 292-302 (2011).

[6] R. Crooks and D. V. Boger, influence of fluid elasticity on drops impacting on dry surfaces, J. Rheol 44,973-996 (2000).

[7] G. German and V. Bertola, Impact of shear-thinning and yield-stress drops on solid surfaces, J. Phys.-Condens. Mat. 21, 375111 (2009).

[8] L.-H. Luu and Y. Forterre, Drop impact of yield-stress fluids, J. Fluid Mech. 632, 301-327 (2009).

[9] M. Guémas, A. G. Marin and D. Lohse, Drop impact experiments of non-Newtonian liquids on microstructured surfaces, Soft Matter 8, 10725-10731 (2012).

[10] J. H. Moon, J. B. Bong and S. H. Lee, Dynamic behaviorof non-Newtonian droplets impinging on solid surfaces, Matls. Trans. 54, 260-265 (2013).

[11] P. Aussillous and D. Quéré, Liquid marbles, Nature 411, 924-927 (2001); Properties of liquid marbles, Proc. R. Soc. A 462, 973-999 (2006).

[12] M. Nicolas, Spreading of a drop of neutrally buoyant suspension, J. Fluid Mech. 545, 271-280 (2005).

[13] I. R. Peters, Q. Xu and H. M. Jaeger, Splashing onset in dense suspension drops, Phys. Rev.Lett. 111, 028301 (2013).

[14] J. O. Marston, M.M. Mansour and S. T. Thoroddsen Phys. Rev. E 88, R010201 (2013).

[15] L. A. Lubbers, Q. Xu, S. Wilken, W. W. Zhang and H. M. Jaeger, Dense suspension splat: monolayer spreading adn hole formation after impact, Phys. Rev. Lett. 113, 044502 (2014).

[16] C. Planchette, A.-L. Biance, O. Pitois and E. Lorenceau, Coalecence of armored interfaces 
under impact, Phys. Fluids 25, 042104 (2013).

[17] J. Koplik and R. Zhang, Nanodrop impact on solid surfaces, Phys. Fluids 25, 022003 (2013).

[18] R. Zhang, S. Farokhirad, T. Lee and J. Koplik, Multiscale liquid drop impact on wettable and textured surfaces, Phys. Fluids 26, 082003 (2014).

[19] N. Ashgriz and J. Y. Poo, Coalescence and separation in binary collisions of liquid drops, J. Fluid Mech. 221, 183-204 (1990).

[20] J. Qian and C. K. Law, J. Fluid Mech. 331, 59-80 (1997).

[21] J. Eggers, J. R. Lister and H. A. Stone, Coalescence of liquid drops, J. Fluid Mech. 401, 293-310 (1999).

[22] I. V. Roisman, C. Planchette, E. Lorenceau and G. Brenn, Binary collisions of drops of immiscible liquids, J. Fluid Mech. 690, 512-535 (2012).

[23] J. D. Paulsen, J. C. Burton, S. R. Nagel, S. Appathurai, M. T. Harris and O. A. Basaran, The inexorable resistance of inertia determines the initial regime of drop coalescence, Proc. Nat. Acad. Sci. USA 109, 6857-6861 (2012).

[24] D. Greenspan and L. F. Heath, Supercomputer simulation of the modes of colliding microdrops of water, J. Phys. D 24, 2121-2123 (1991).

[25] J. Koplik and J. R. Banavar, Molecular structure of the coalescence of liquid interfaces, Science 257, 1664-1667 (1992).

[26] M. Svanberg, L. Ming, N. Marković and J. B. C. Pettersson, Collision dynamics of large water clusters, J. Chem. Phys. 108, 5888-5897 (1998).

[27] L. Zhao and P. Choi, Molecular dynamics simulation of the coalescence of nanometer-sized waterdroplets in n-heptane, J. Chem. Phys. 120, 1935-1942 (2004).

[28] R.-R. Juang, Y.-M. Lee, C.-H. Chang, J.-S. Wu, Y.-L. Hsu and S.-W. Chau, Parallel molecular dynamics simulation of head-on collision of two nanoscale droplets with low relative speed, J. Comput. Theor. Nanosci. 6, 46-53 (2009).

[29] J.-C. Pothier and L. J. Lewis, Molecular dynamics study of the viscous to inertial crossoever in nanodroplet coalescence, Phys. Rev. E 85, 115447 (2012).

[30] E. Kompinski and E. Sher, Experimental comparisons between droplet-droplet collision and single-droplet impacts on a solid surface, Atomization and Sprays 19, 409-423 (2009).

[31] M. Gross, I. Steinbach, D. Raabe and F. Varnik, Viscous coalescence of droplets: A lattice Boltzmann study, Phys. Fluids 25, 052101 (2013). 
[32] L.Xu, W.W. Zhang and S. R.Nagel, Drop splashing on a dry smooth surface, Phys. Rev.Lett. 94, 184505 (2005).

[33] M. P. Allen and D. J. Tildesley, Computer Simulation of Liquids (Oxford, Clarendon Press, 1987). 

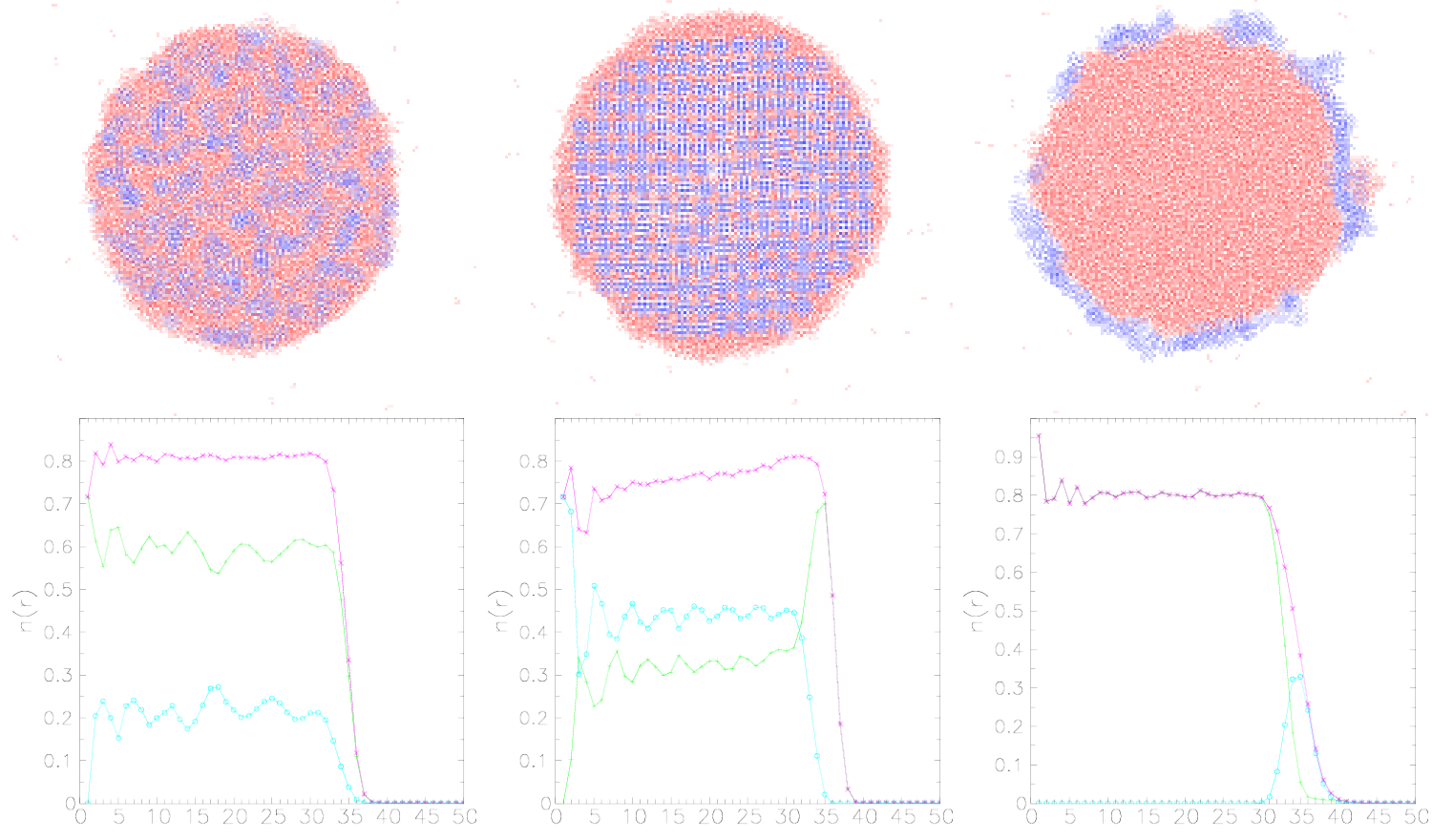

FIG. 1. Top row, left to right: snapshots of a $10 \sigma$ section through the center of the $21 \%, 42 \%$ and marble drops. Fluid and particle atoms are shown as red (light) and blue (dark) dots. Bottom row, left to right: radial density plots of the same three drops. Particle density is shown in cyan ("o"), fluid density in green ("+") and total density in magenta ("x"). 
liquid
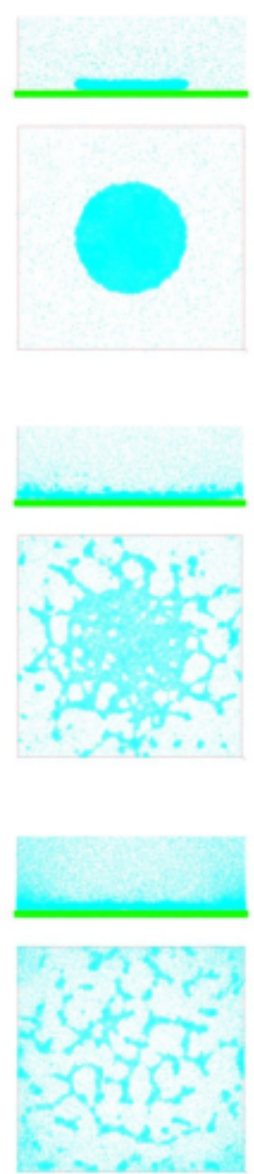

$21 \%$
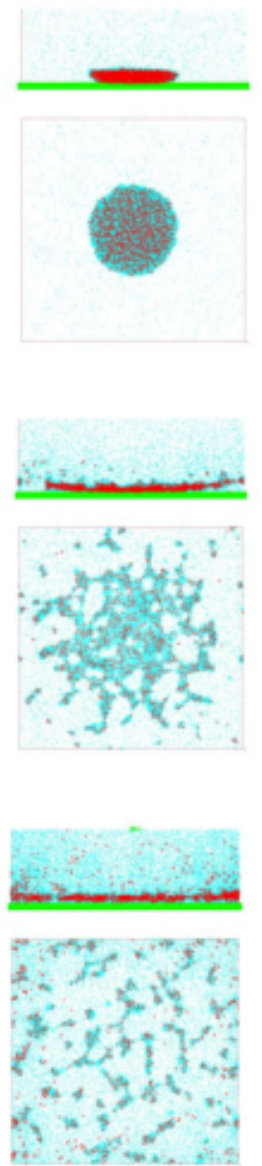

$42 \%$
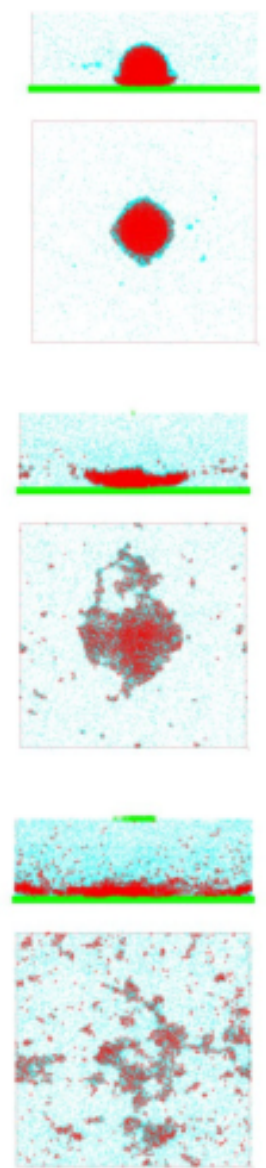

marble
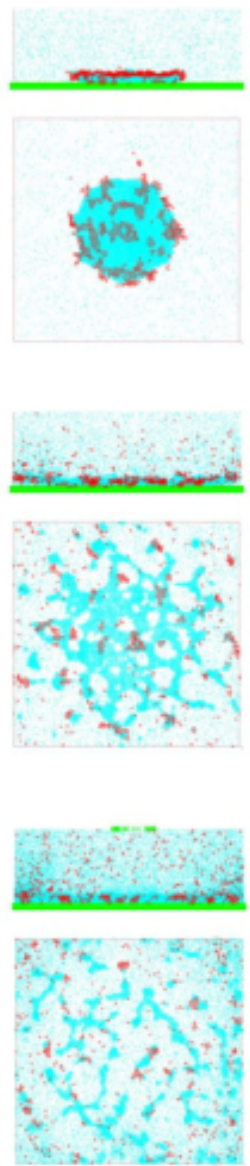

FIG. 2. Snapshots of different drops impacting a non-wetting surface at time $100 \tau$. Columns, left to right: pure liquid, $21 \%$ suspension, $42 \%$ suspension and liquid marble. Rows, top to bottom: initial velocities $u_{0}=1,2,3 \sigma / \tau$. Fluid and solid atoms shown in cyan and red, respectively. 
liquid
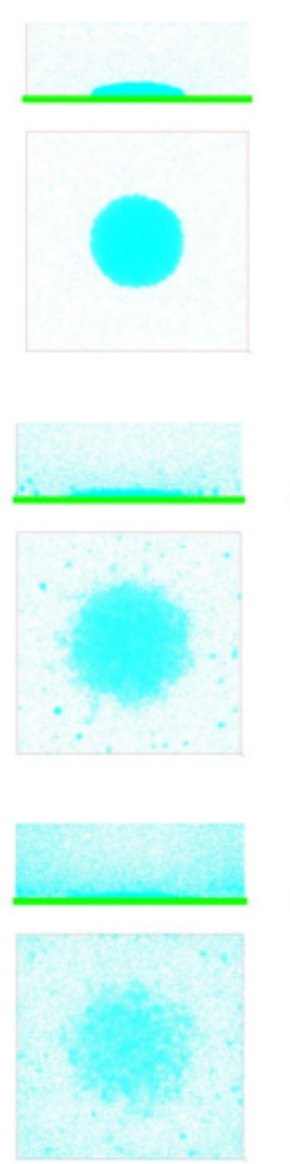

$21 \%$
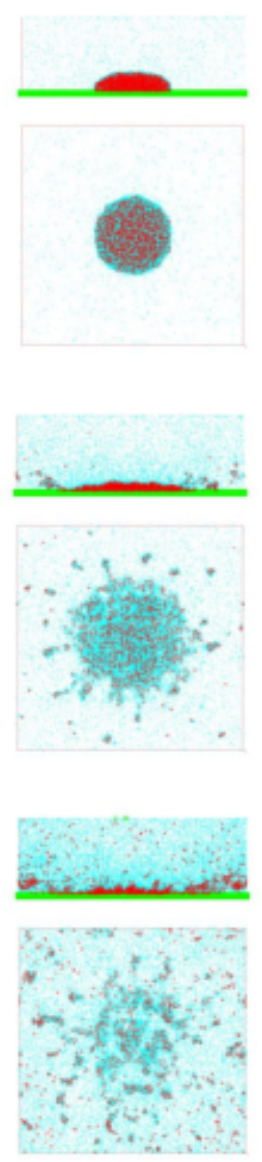

$42 \%$
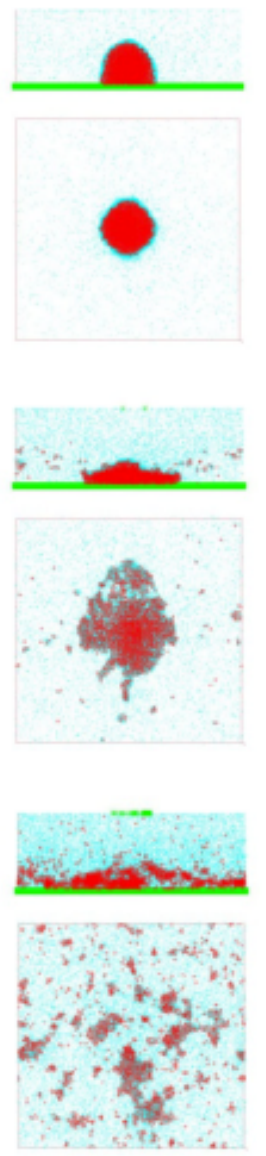

marble
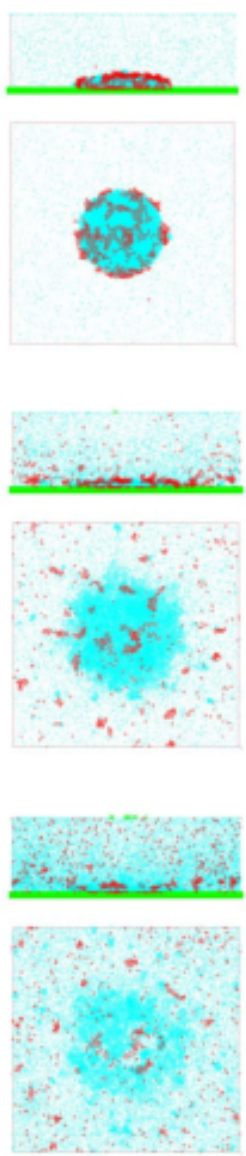

FIG. 3. Side view plus top view snapshots of different drops impacting a wetting surface at time $100 \tau$. Columns, left to right: pure liquid, $21 \%$ suspension, $42 \%$ suspension and liquid marble. Rows, top to bottom: initial velocities $u_{0}=1,2,3 \sigma / \tau$. Fluid and solid atoms shown in cyan and red, respectively. 


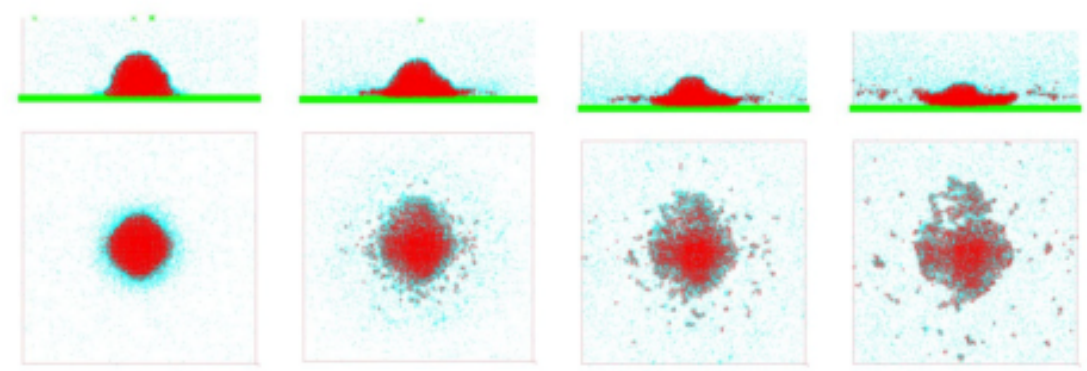

FIG. 4. Impact of a $42 \%$ suspension drop on a non-wetting surface at velocity $u_{0}=2 \sigma / \tau$, at times (left to right) 20, 35, 50 and $75 \tau$. For $100 \tau$ see Fig. 2, 
liquid
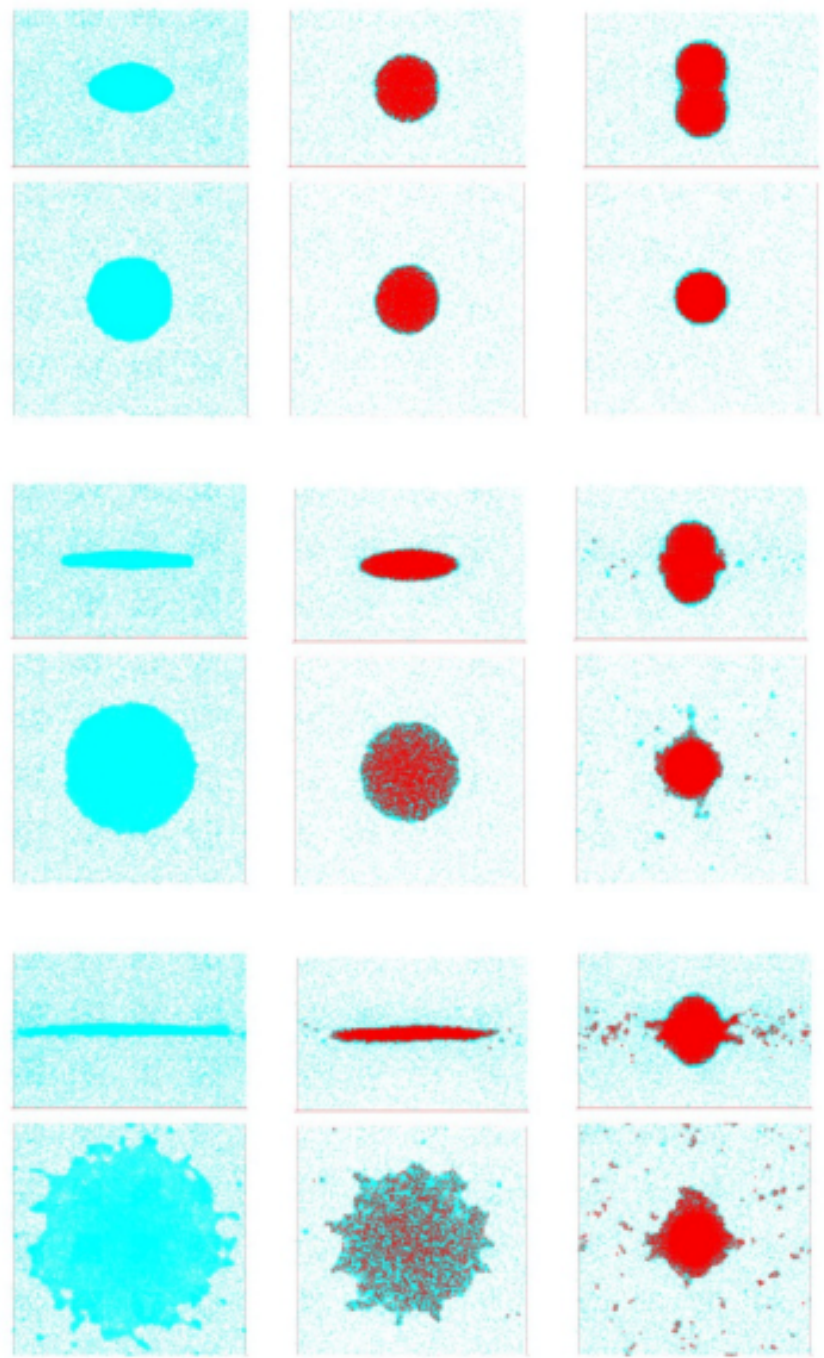

$42 \%$
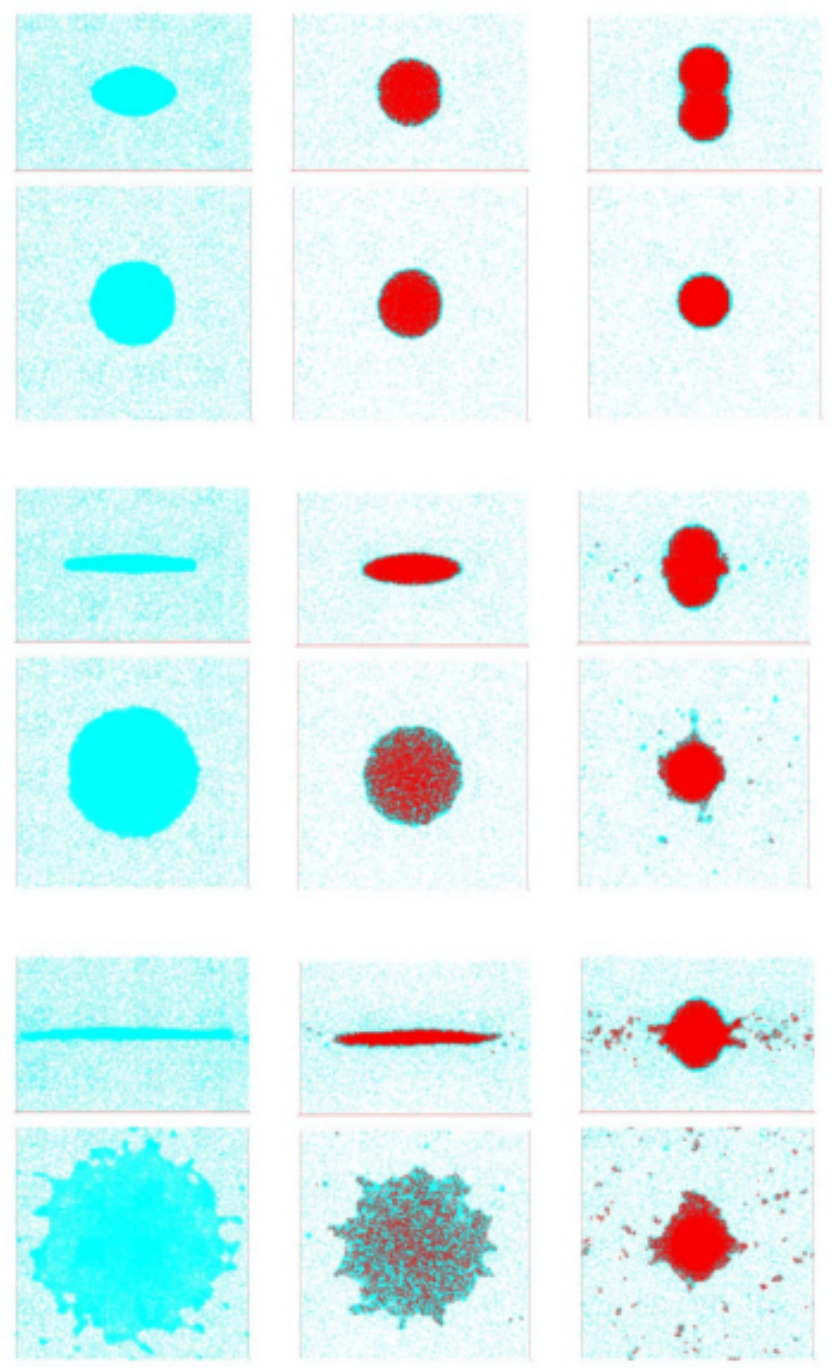

$21 \%$
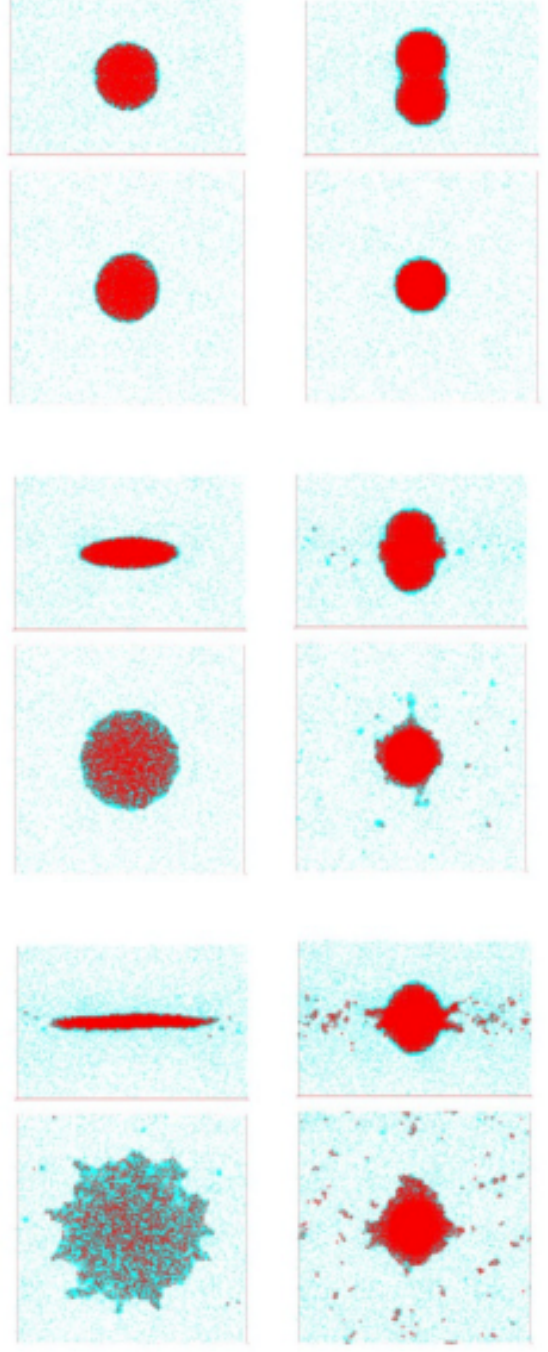

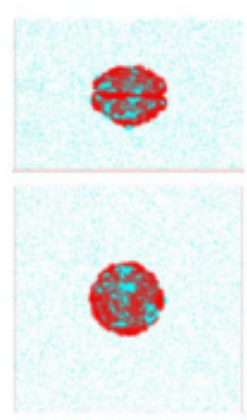

marble
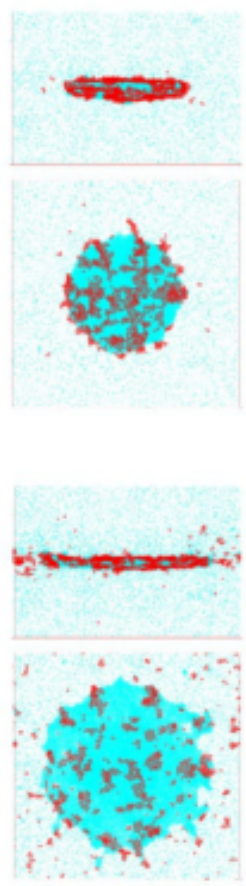

FIG. 5. Snapshots of head-on collisions of drops of the same material at time $100 \tau$. Columns, left to right: pure liquid, $21 \%$ suspension, $42 \%$ suspension and liquid marble. Rows, top to bottom: initial relative velocities $u_{0}=1,2,3 \sigma / \tau$. Fluid and solid atoms shown in cyan and red, respectively. 


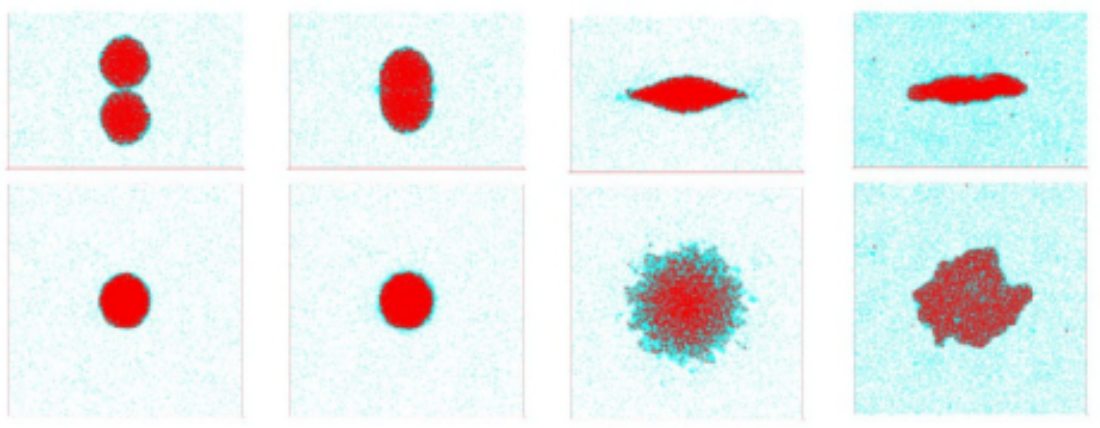

FIG. 6. Collision of two identical drops of a $21 \%$ suspension at relative velocity $3 \sigma / \tau$ at times (left to right) $10,20,50$ and $500 \tau$; the $100 \tau$ snapshot is included in Fig. 5 . 
liquid
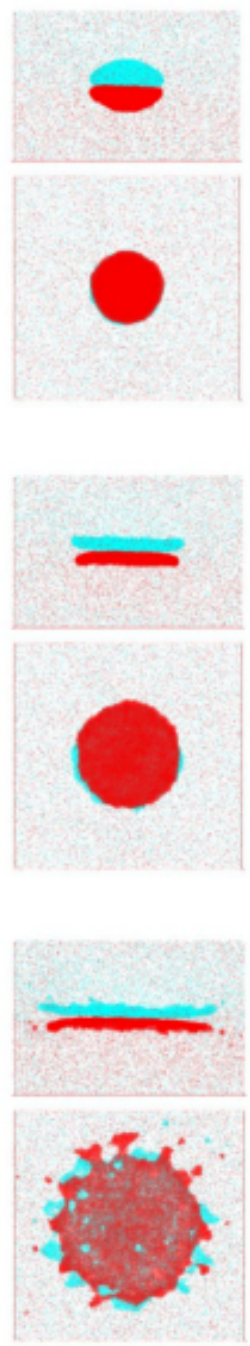

$21 \%$
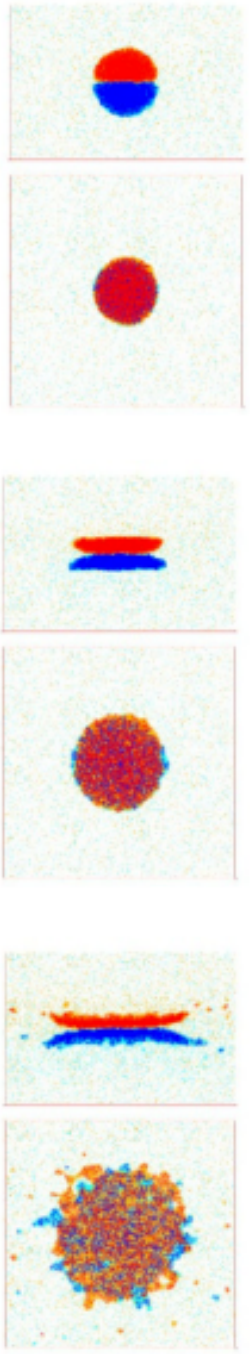

$42 \%$
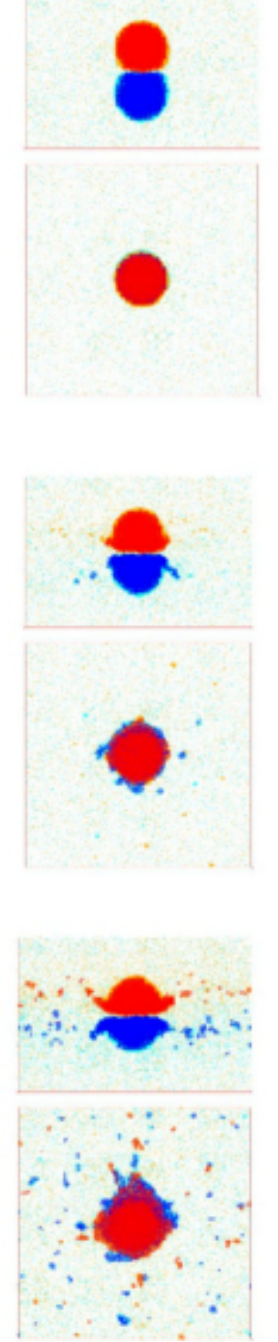

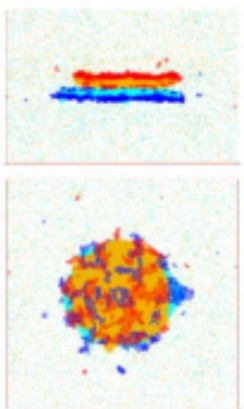

marble
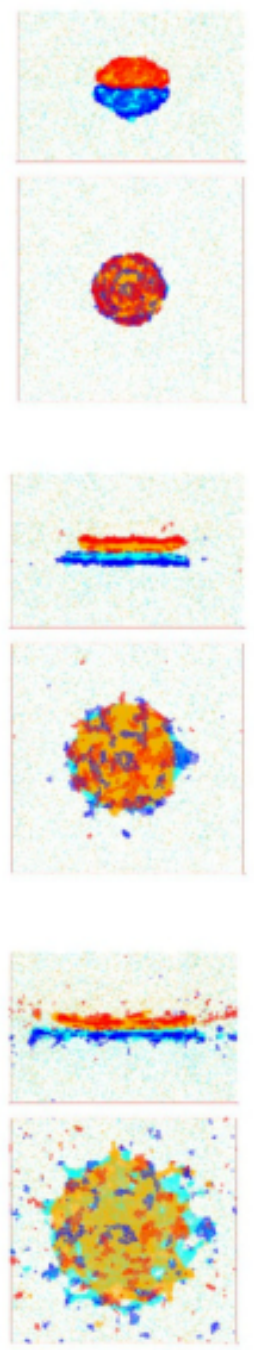

FIG. 7. Snapshots of head-on collisions of drops of the "opposite" material, as define in the text, at time $100 \tau$. Columns, left to right: pure liquid, $21 \%$ suspension, $42 \%$ suspension and liquid marble. Rows, top to bottom: initial relative velocities $u_{0}=1,2,3 \sigma / \tau$. Fluid and solid atoms shown in cyan and red, respectively. 


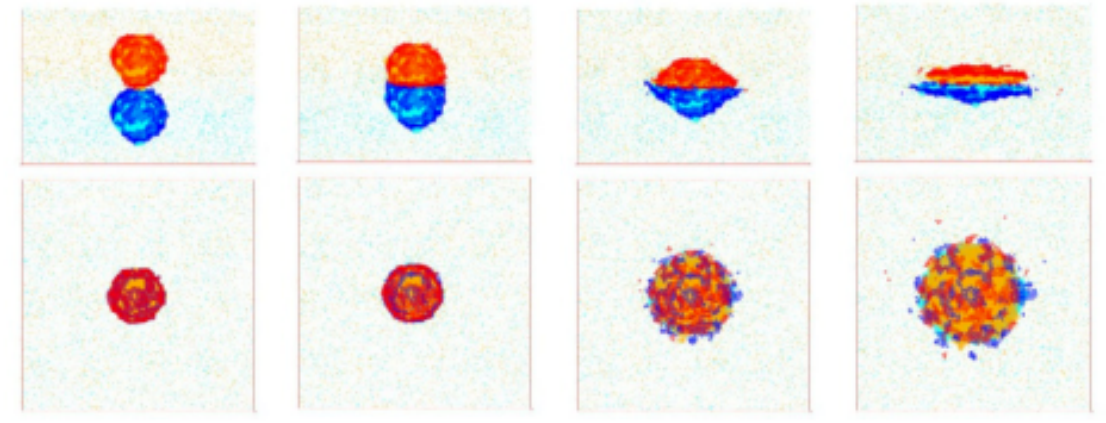

FIG. 8. Collision of two "opposite" drops of a liquid marble at relative velocity $2 \sigma / \tau$ for times (left to right) 15, 30, 50 and $75 \tau ; 100 \tau$ is included in Fig. 7 . 


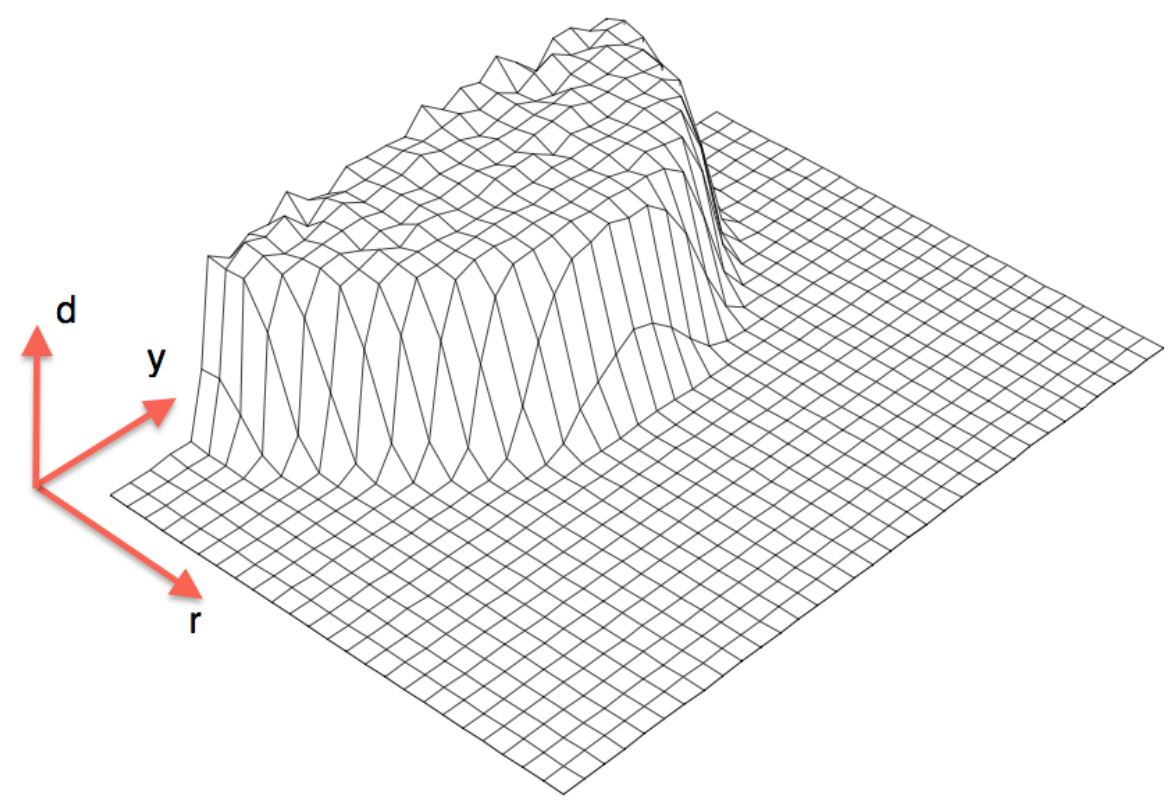

FIG. 9. Surface elevation plot of the two-dimensional density field $d(r, y)$ of an equilibrated $21 \%$ suspension drop, in cylindrical coordinates averaged over azimuthal angle, where $y$ is the height above the surface and $r$ is distance from the $y$-axis. 
Total de nsity
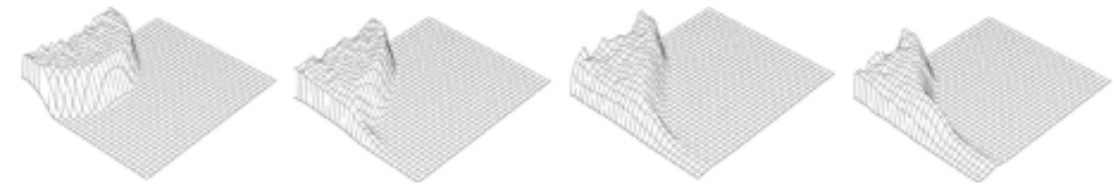

potential ene rgy
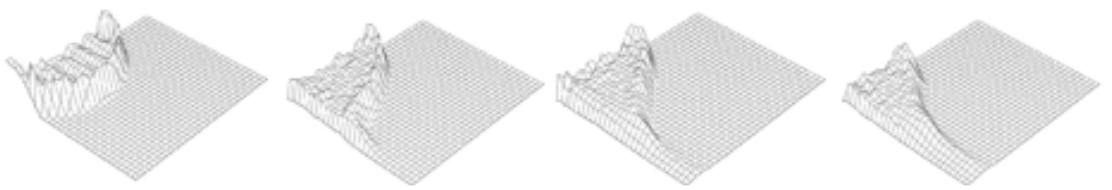

fluid kinetic e nergy
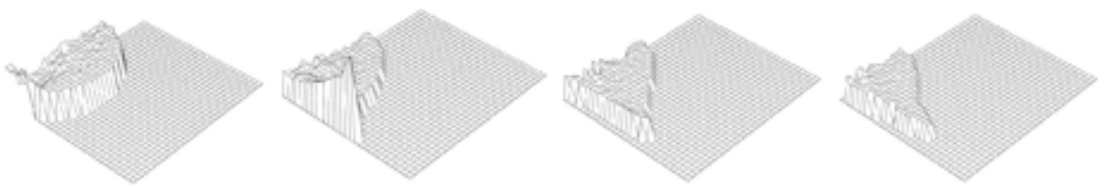

particle kine tic energy
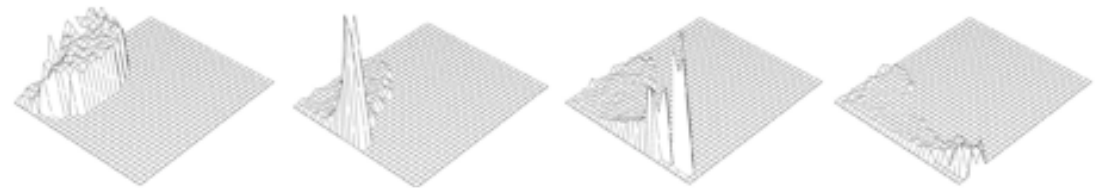

te mpera ture
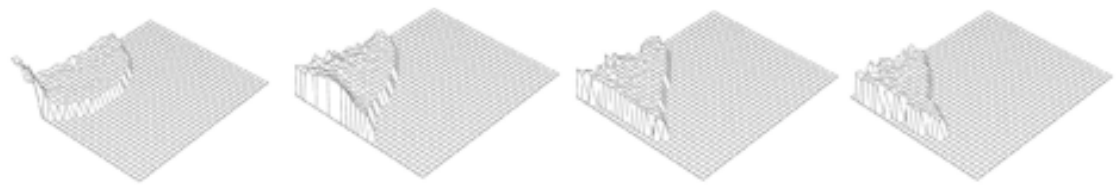

FIG. 10. Surface elevation plot of the two-dimensional density and energy fields arising in the surface impact of a $42 \%$ suspension drop on a non-wetting surface. The format is that of Fig. 9 and the corresponding snapshots of the impact are given in Fig. 4Left to right: times 10, 20, 30 and $50 \tau$. Top to bottom: total density, potential energy density, fluid kinetic energy, particle kinetic energy and temperature. The maximum heights in the left-hand frames are density: $0.946 \sigma^{-3}$, potential energy: $5.66 \epsilon$, fluid kinetic energy: $5.49 \epsilon$, particle kinetic energy: $75.35 \epsilon$ and temperature $3.02 \epsilon / k_{B}$. 


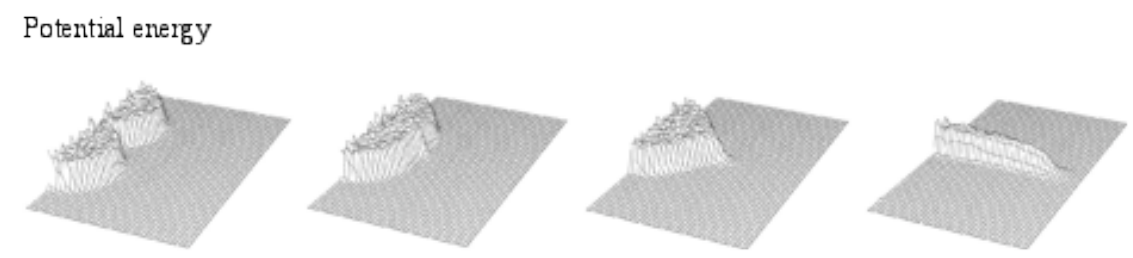

fluid kinetic e nergy
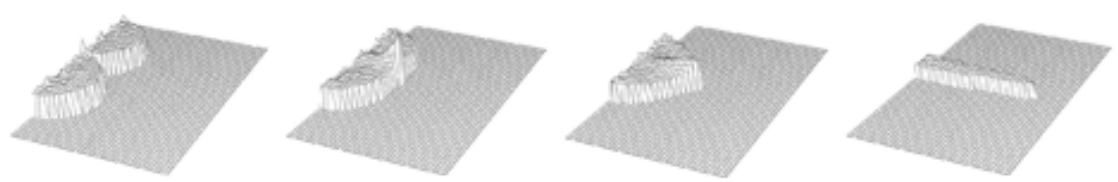

particle kine tic energy
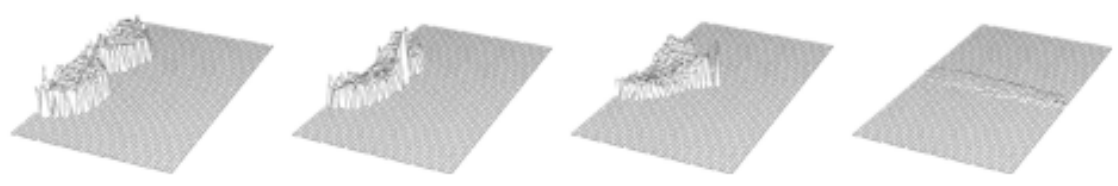

FIG. 11. Surface elevation plot of the two-dimensional density fields arising in the collision of two $21 \%$ suspension drops at velocity $3.0 \sigma / \tau$ The format is that of Fig. 9 and the corresponding snapshots of the impact are given in Fig. 6Left to right: times 10, 20, 30 and 100 $\tau$. Top to bottom: potential energy density, fluid kinetic energy and particle kinetic energy. The maximum heights in the left-hand frames are potential energy: $5.01 \epsilon$, fluid kinetic energy: $3.92 \epsilon$ and particle kinetic energy: $61.2 \epsilon$. 

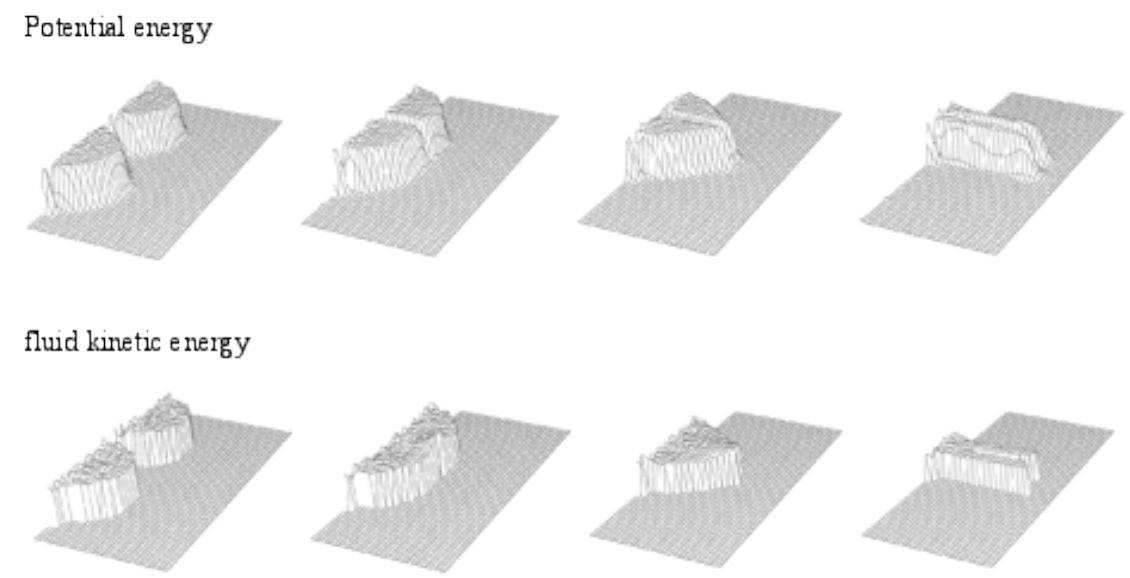

particle kine tic energy
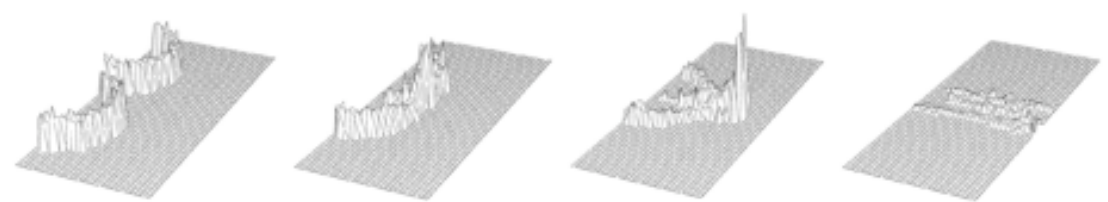

FIG. 12. Surface elevation plot of the two-dimensional density fields arising in the collision of two "opposite" liquid marble drops at velocity $2.0 \sigma / \tau$. The format is that of Fig. 9 and the corresponding snapshots of the impact are given in Fig. 8 Left to right: times 10, 30, 50 and $100 \tau$. Top to bottom: potential energy density, fluid kinetic energy and particle kinetic energy. The maximum heights in the left-hand frames are potential energy: $5.11 \epsilon$, fluid kinetic energy: $2.06 \epsilon$ and particle kinetic energy: $24.4 \epsilon$. 

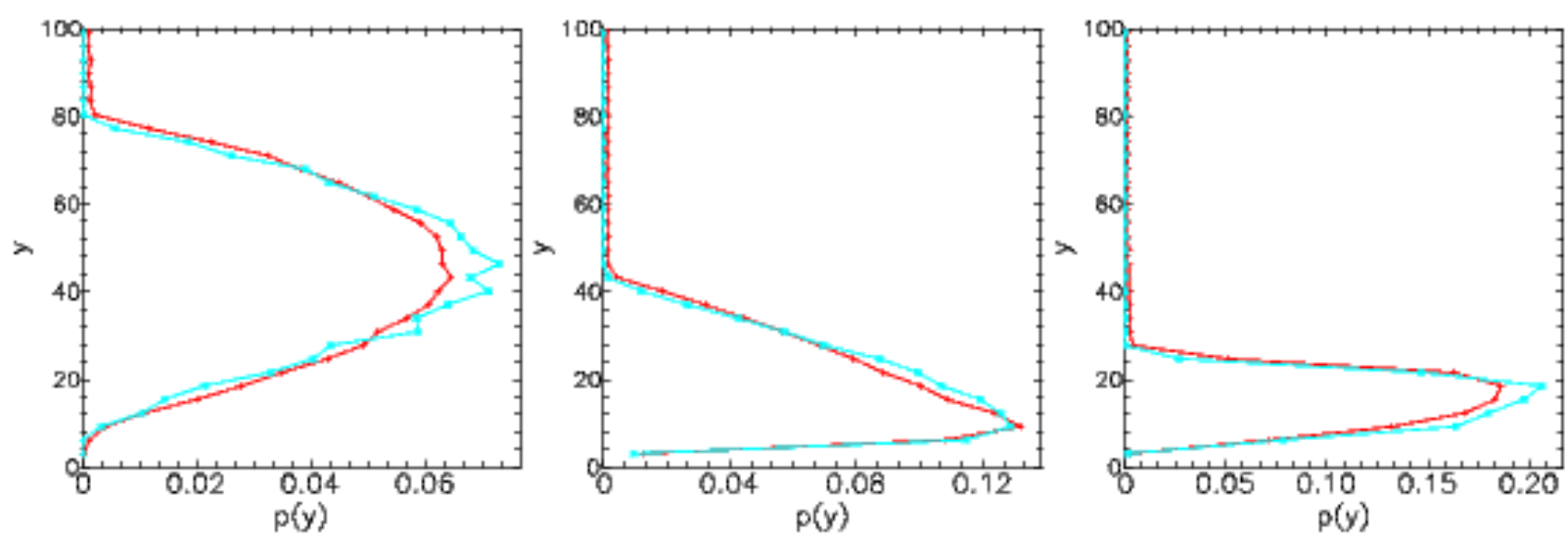

FIG. 13. Probability density of fluid (red + ) and particles (cyan *) as a function of height $y$ for the impact of a $21 \%$ suspension drop on a non-wetting wall at velocity $2 \sigma / \tau$, at times (left to right) 10,50 and $100 \tau$.
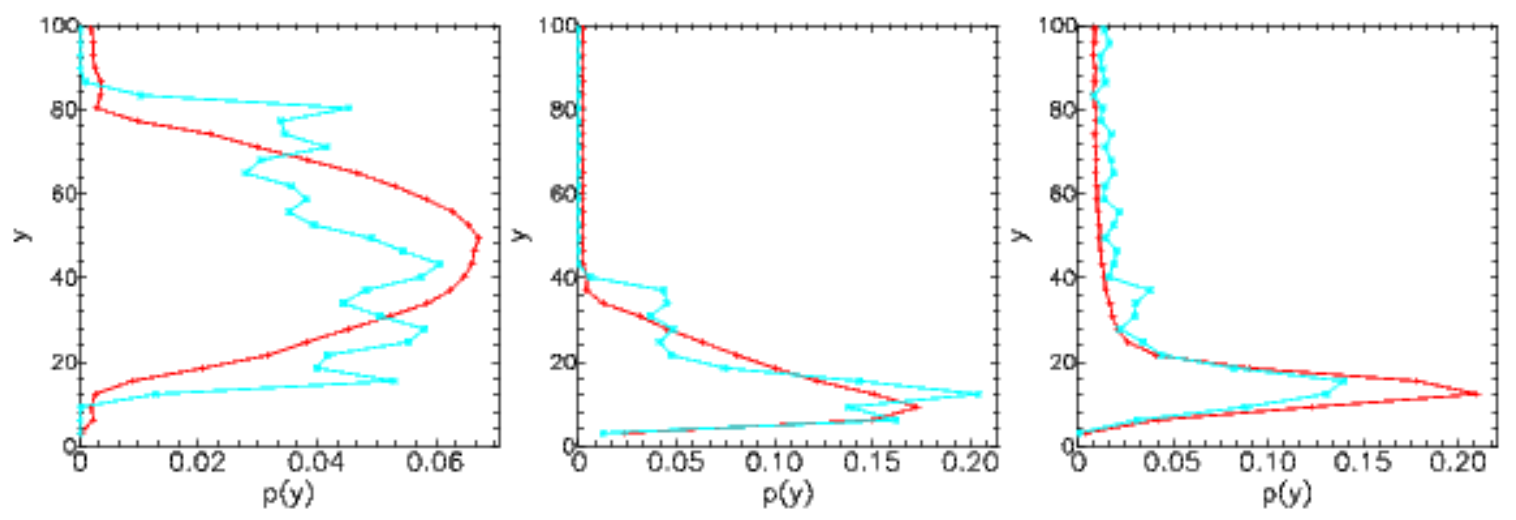

FIG. 14. Probability density of fluid (red + ) and particles (cyan $\left.{ }^{*}\right)$ as a function of height $y$ for the impact of a liquid marble drop on a non-wetting wall at velocity $3 \sigma / \tau$, at times (left to right) 10,50 and $100 \tau$. 

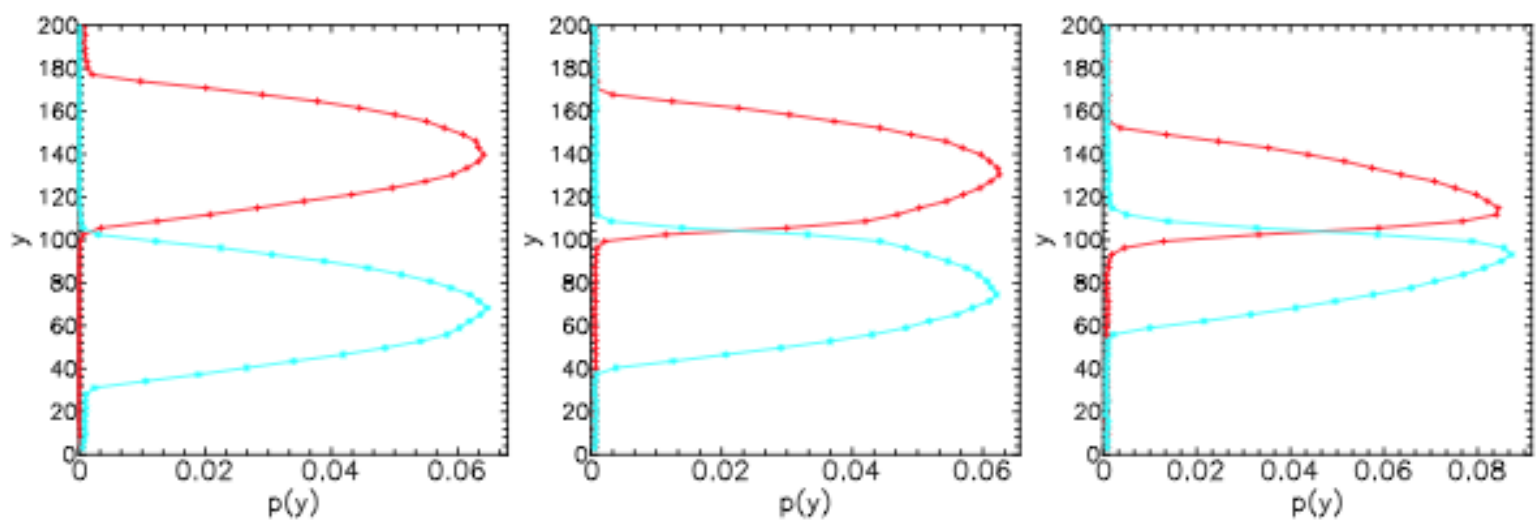

FIG. 15. Probability density of fluid initially in the upper $($ red +$)$ and lower $($ cyan $*)$ drops as a function of height $y$ for the coalescence of two identical liquid drops at times (left to right) 10, 200 and $500 \tau$.
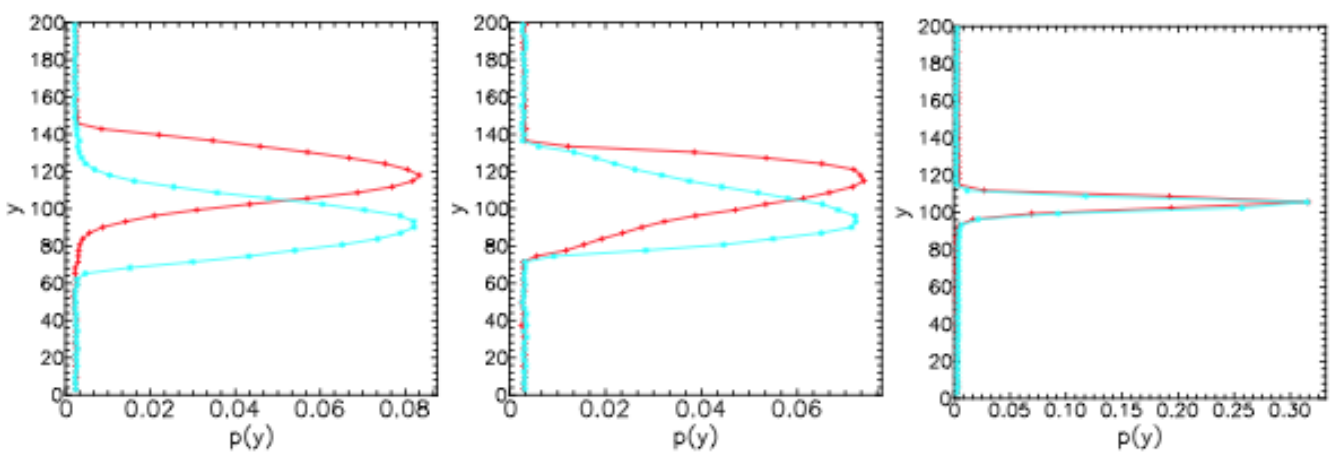

FIG. 16. Probability density of fluid initially in the upper $($ red +$)$ and lower (cyan *) drops as a function of height $y$ for collision of two identical liquid drops at time $500 \tau$ for relative velocities (left to right) 1,2 and $3 \sigma / \tau$. 


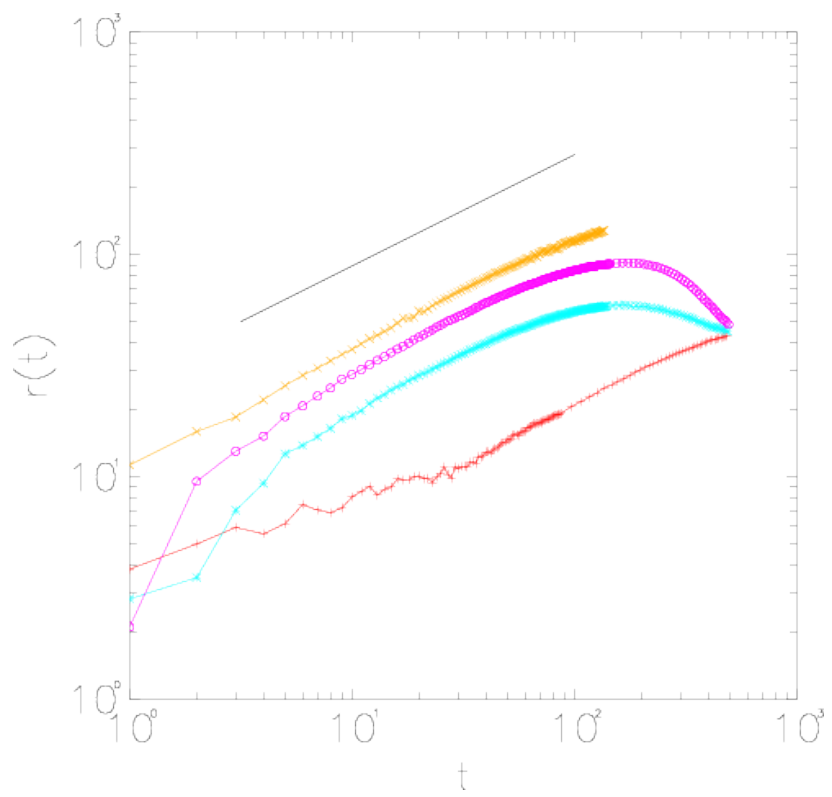

FIG. 17. Time dependence of the neck radius of two merging drops at various velocities, where $t=1$ is the earliest time at which a neck can be identified. Bottom to top: neck radius for collisions at relative velocities 0 (free coalescence, red), 1 (blue), 2 (magenta) and $3 \sigma / \tau$ (orange). The solid black line at the top shows $r(t) \sim t^{1 / 2}$ for comparison.
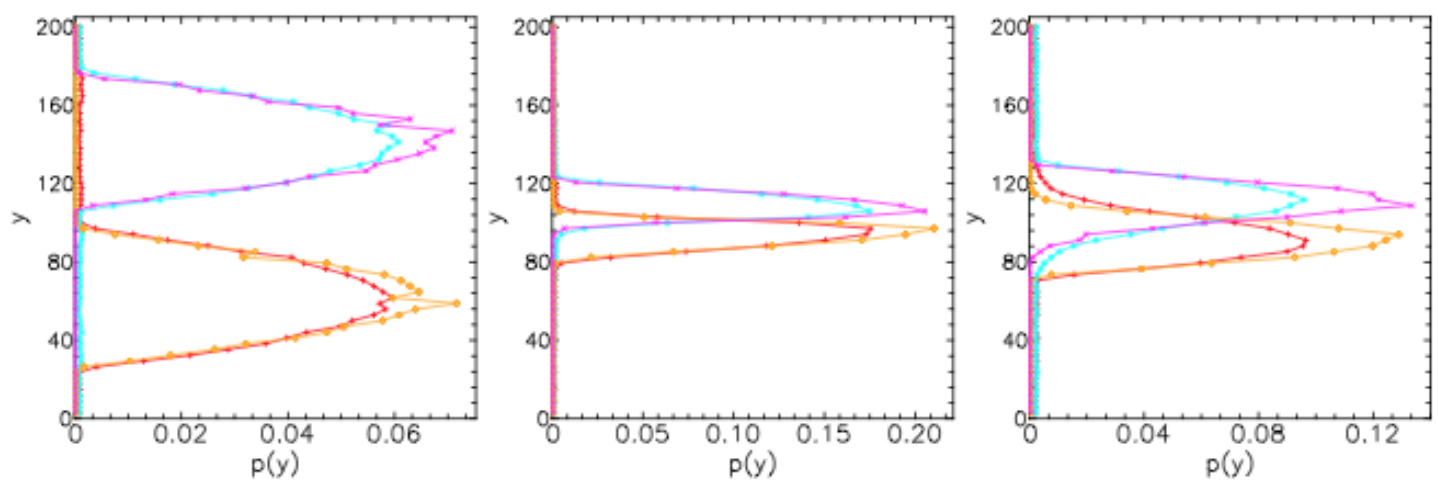

FIG. 18. Probability density of fluid and particles initially in the upper drop (cyan * and magenta $\mathrm{o}$, respectively) and likewise for the lower drop (red + for fluid and orange $\mathrm{x}$ for particles) for the collision of two $21 \%$ suspension drops of the same material, at relative velocity $2 \sigma / \tau$, as a function of height $y$, at times (left to right) 10, 100 and $500 \tau$. 

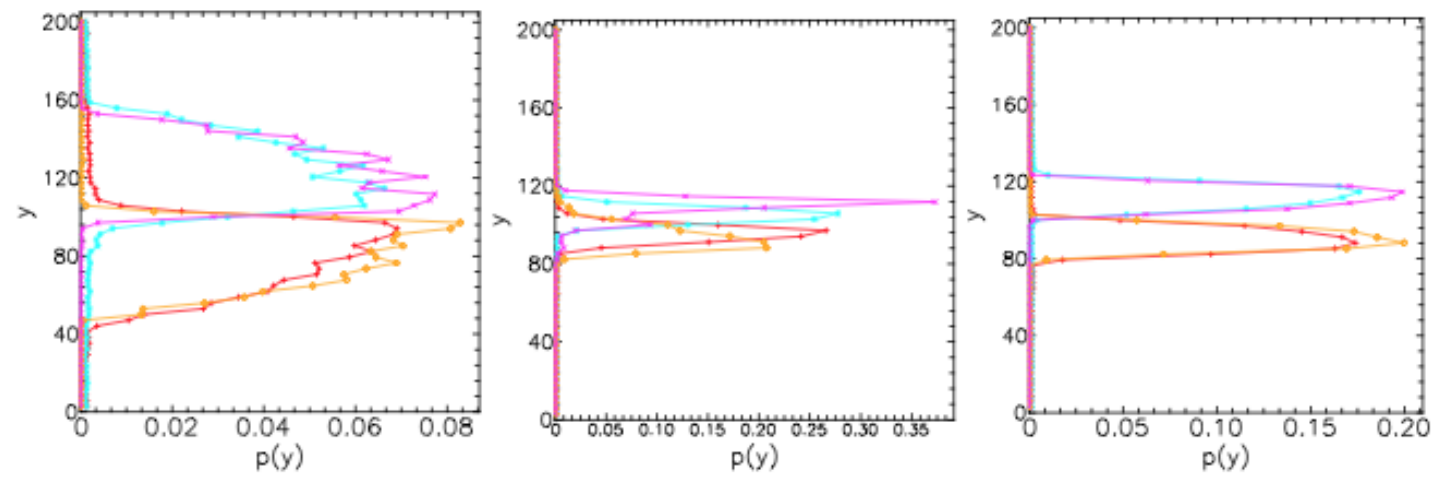

FIG. 19. Probability density of fluid and particles initially in the upper drop (cyan ${ }^{*}$ and magenta o, respectively) and likewise for the lower drop (red + for fluid and orange $\mathrm{x}$ for particles) for Left: two $42 \%$ suspension drops, Middle: liquid marble and Right: "opposite" $21 \%$ suspension drops, all at relative velocity $2 \sigma / \tau$ at time $100 \tau$. 